\title{
A Phase Field - Finite Element Approach to Model the Interaction Between Phase Transformations and Plasticity in Shape Memory Alloys
}

\author{
Harshad M. Paranjape ${ }^{a^{*}}$, Sivom Manchirajua, Peter M. Andersona \\ aMaterials Science and Engineering, The Ohio State University, 2041 College Rd, Columbus, OH 43210 \\ USA \\ paranjape.2@osu.edu (614) 961-7796, manchiraju.1@osu.edu, anderson.1@osu.edu (614) 292-0176 \\ * Corresponding author
}

\begin{abstract}
The coupling between phase transformations and plasticity in shape memory alloys (SMAs) is studied by developing a finite element framework in which the constitutive relation captures both phase transformations at the martensite correspondence variant (CV) scale and rate-dependent crystal plasticity in austenite. Load-free and load-biased thermal cycling simulations involving a model cubic-to-tetragonal transformation system are carried out to study how slip in austenite can affect the resulting martensite microstructure. Three key questions are answered. First, where does austenite slip predominantly occur during phase transformation? Second, at what stage during a thermal cycle is plastic deformation most pronounced? Third, what is the effect of plastic deformation on measurable parameters like transformation temperature and subsequent transformation microstructure? The model can also be generalized to study the coupling between phase transformations, twining, and slip.
\end{abstract}

Keywords: A. Phase Transformation; B. Crystal plasticity; B. Constitutive behavior; C. Finite elements; Shape memory alloys

\section{Introduction}

Shape memory alloys (SMAs) have found wide use in actuators and medical implants due to their remarkable shape memory effects and superelasticity (Mohd Jani et al., 2014). An underlying martensitic phase transformation is responsible for these properties. The transformation between a high temperature phase (austenite) and a low temperature phase (martensite) can be initiated by a change in temperature and/or external stress. This phase change typically produces a strain of several \% that can be recovered when the temperature and/or external stress are reversed. In many SMA systems, this process is accompanied by plasticity (slip) (Benafan et al., 2014; Manchiraju et al., 2011; Norfleet et al., 2009). This poses a two-fold problem to commercial applications. First, a nonrecoverable strain can accumulate during thermal or stress cycling, leading to changes in component geometry. This phenomenon is commonly referred to as functional fatigue. Second, the defect accumulation can assist in crack nucleation and eventual failure of the SMA assembly. This is referred to as structural fatigue (Eggeler et al., 2004). A detailed understanding of the coupling between phase transformation and slip is needed to mitigate these fatigue mechanisms. 
Recent experimental work suggests that austenite slip is one of the mechanisms by which local incompatibility at the austenite-martensite interface is accommodated. Experiments on NiTi polycrystals show evidence for austenite dislocations generated during stress or thermal cycling (Delville et al., 2011; Pelton et al., 2012). However these observations are qualitative, primarily because the intergranular constraints in polycrystals lead to a complex martensite microstructure and slip distribution. Single crystal experiments have characterized in detail the coupling between slip and phase transformation in SMAs. Hamilton et al. report dislocation arrays at the interface between austenite and martensite in solutionized Ti-50.1at\% Ni single crystals thermally cycled under a biasing stress along the [ $\left.\begin{array}{lll}1 & 1 & 1\end{array}\right]$ axis (Hamilton et al., 2004). They show tangled planar dislocation arrays in the austenite phase. In principle, this plasticity could be driven by the macroscopic applied stress because the Schmid factor for plasticity in [1 111 1]-oriented NiTi austenite is large. However, transmission electron microscope (TEM) studies coupled with crystallographic modeling of NiTi single crystal micro-pillars by Norfleet $e t$ al. and Bowers $e t$ al. correlate the formation of stress-induced martensite correspondence variant (CV) pairs with activity on (1 0 -

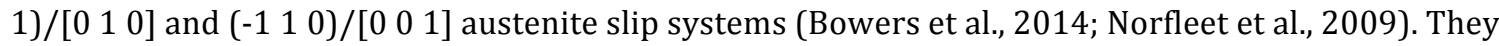
hypothesize that the large stresses generated by the local incompatibility between austenite and monoclinic martensite CV pairs are the source of slip in austenite. The slip systems observed in these studies are predicted to have the largest resolved shear stress due to the stress generated by nearby austenite-martensite interfaces. A similar observation of dislocation loops at an austenite-FCC martensite interface is made by Tseng et al. in superelastically-deformed Fe-34\% Mn-15\%Al-7.5\% Ni (at.\%) single crystals. Here, the loop spacing is observed to be comparable to the CV twin width (Tseng et al., 2015). Other experimental work demonstrates that transformation-induced austenite slip can influence the transformation temperatures (Pelton et al., 2012) and transformation initiation stress (Polatidis et al., 2015). Although the experiments provide clear evidence of the interaction between phase transformation and slip, it is attractive to develop a mathematical model to provide a predictive connection between the two mechanisms.

Phenomenological models with internal variables and physics-based micromechanical models have been used extensively to study both independent and coupled phase transformation and slip in SMAs (for a broad overview see Cisse et al., 2016). Representative phenomenological models include work by Auricchio et al. (Auricchio et al., 2008; Auricchio and Petrini, 2004), Zaki and Moumni (Zaki and Moumni, 2007), Thamburaja (Thamburaja, 2010) and Stebner and Brinson (Stebner and Brinson, 2013). These phenomenological models track the total martensite volume fraction rather than the evolution of individual martensite variants. Their key advantages are computational efficiency and the ability to easily calibrate parameters to specific experiments. Among the physics-based micromechanical models, Thamburaja and Anand (Thamburaja and Anand, 2001) captured phase transformation at the martensite habit plane scale. Subsequently, Thamburaja et al. developed micromechanical models for detwinning and reorientation of martensite CVs (Pan et al., 2007; Thamburaja, 2005). A variety of models simulate the interaction between various inelastic mechanisms at the micro length scale. Manchiraju and Anderson coupled phase transformation at the martensite CV pair scale and continuum-based plasticity in austenite by computing the local redistribution of stresses caused by each mechanism (Manchiraju and Anderson, 2010). Although these models simulate the evolution of the crystallographic variants of martensite, the spatial information about the martensite microstructure at the grain scale is not captured. Yu et al. recently developed micromechanics-informed models that couple multiple inelastic mechanisms (Yu et al., 2015a, 2015b, 2013). These were used to study the effect of multi-axial and cyclic loading on the interaction between slip and transformation. At the polycrystalline length scale, Richards et al. systematically studied transformation-slip coupling (Richards et al., 2013). They viewed slip as a 
bridging mechanism in the propagation of phase transformation through various grains during loading. Recently, the phase field method has been used extensively to study the interaction between inelastic mechanisms in SMAs at the martensite microstructure length scale.

The initial work of Wang and Khachaturyan (Wang and Khachaturyan, 1997) and Artemev et al. (Artemev et al., 2001) introduced phase field models of phase transformations in metallic systems. Levitas et al. studied the interaction between phase transformation and plasticity at the scale of martensite CVs (Javanbakht and Levitas, 2015; Levitas and Javanbakht, 2015, 2013). Their models incorporate finite deformations and energy landscapes between austenite and martensite that account for changes in modulus during transformation and interaction with dislocations. However, they present only 2D results. Yamanaka et al. and Malik et al have adopted a continuum scale model of slip (Malik et al., 2013; Yamanaka et al., 2010, 2008) and a similar approach is used by Kundin et al. (Kundin et al., 2015, 2011). Their plasticity law includes various mechanisms of slip evolution such as dislocation generation and annihilation and the transformation law models a cubic-totetragonal transformation system with three CVs. A key result is that the slip system with the largest interaction stress with the martensite variants exhibits the largest plastic strain. However, these approaches rely on infinitesimal strain measures and Fourier transform-based solvers. An infinitesimal strain formulation for SMA has been shown to overestimate the martensite volume fraction and predict an overly stiff rotational response (Christ and Reese, 2008; Reese and Christ, 2008).Further, the authors are not aware of a systematic temporal and spatial study of the interaction between the inelastic mechanisms in 3D at the martensite CV scale in SMAs.

We address both of these issues by introducing a new model that couples martensite CV scale phase transformation with continuum-scale slip in austenite, within a large-deformation formalism. These results can be compared directly with experimental techniques like TEM and high energy X-ray diffraction. The coupled constitutive law is developed in Section 2. We present a systematic study of the interaction between phase transformation and plasticity to reveal the effect of this interaction on measurable parameters like transformation temperatures. We attempt to answer the questions raised by TEM-based experiments: Is there a spatial correlation between the slip in austenite and the martensite microstructure? Do the transformation temperatures change after slip activity in austenite? How are the spatial features of the martensite microstructure and slip related? Such correlations typically are difficult to observe experimentally. The simulations used to perform this study are described in Section 3. The results are presented in Section 4 and discussed in Section 5. The large-deformation formalism and FEM-based implementation enable simulation of the interaction between phase transformation, twinning and slip in a variety of materials.

\section{Phase Transformation and Crystal Plasticity Constitutive Law}

A constitutive law combining the deformation mechanisms of anisotropic elasticity, thermal expansion, austenite-martensite phase transformation, and slip in austenite is described in this section. The aim of this law is to calculate the increments in stress, martensite microstructure and slip activity at a material point given the current austenite and martensite microstructure as well as the current and proposed increment in deformation gradient. The microstructure includes the spatial distribution and local crystallographic orientation of austenite and correspondence variants of martensite as well as the anisotropic elastic and inelastic deformation arising from the phase transformation and any slip activity on austenite slip systems. To achieve this, a rate-dependent phase transformation law is constructed using a phase field formalism and a rate-dependent crystal plasticity law for austenite is incorporated. 
Consider an infinitesimal vector $\boldsymbol{d} \mathbf{X}$ in an undeformed configuration. This vector is deformed into $\boldsymbol{d} \boldsymbol{x}$ by elastic deformation, slip and transformation. The correspondence between the undeformed and deformed vector is described by $\boldsymbol{d} \boldsymbol{x}=\mathbf{F} \boldsymbol{d} \boldsymbol{X}$, where $\mathbf{F}$ is the deformation gradient. Following Lee (Lee, 1969), $\mathbf{F}$ is multiplicatively decomposed into the elastic (Fe) and the inelastic (Finel) parts according to

$$
\mathbf{F}=\mathbf{F}^{\mathrm{e}} \mathbf{F}^{\text {inel }}
$$

In the numerical implementation of constitutive laws, a time rate of deformation gradient ( $\left.\dot{\mathbf{F}}^{\text {inel}}\right)$ is typically specified. This can be written as

$$
\dot{\mathbf{F}}^{\text {inel }}=\mathbf{F}^{\text {inel }} \mathbf{L}^{\text {inel }}
$$

The inelastic velocity gradient ( $\mathbf{L}^{\text {inel }}$ ) is approximated by summing the contributions from $t=1$ to $N_{t}$ martensite correspondence variants plus those arising from $t=1$ to $N_{s}$ slip systems in austenite.

$$
\dot{\mathrm{L}}^{\text {inel }}=\sum_{t=1}^{N_{t}} \dot{v}_{t} \mathbf{U}_{t}^{\text {trans }}+v_{A} \sum_{t=1}^{N_{s}} \dot{\gamma_{t}} \mathbf{S}_{t}^{\text {slip }}
$$

Here $\dot{v}_{t}$ is the rate of change of volume fraction $v_{t}$ of the $t^{\text {th }}$ correspondence variant (CV) and $\dot{\gamma}_{t}$ is the rate of slip activity on slip system $t$ in the austenite phase. Eqn. 3 couples the two inelastic mechanisms because both of the inelastic rates are driven by the same stress as shown in Sections 2.1 and 2.2. The transformation stretch matrix $\mathbf{U}_{t}^{\text {trans }}$ specifies the lattice deformation introduced by the phase transformation from austenite to the $t^{\text {th }}$ martensite CV and $\mathbf{S}_{t}^{\text {slip }}=\boldsymbol{b}_{t}^{\text {slip }} \otimes \boldsymbol{m}_{t}^{\text {slip }}$ is the deformation gradient produced by a unit amount of slip activity on austenite slip system $t$ with unit slip direction $\boldsymbol{b}_{t}^{\text {slip }}$ and unit slip plane normal $\boldsymbol{m}_{t}^{\text {slip }}$. The austenite volume fraction is defined by $v_{A}=\mathbf{1}-\sum_{t}^{N_{t}} v_{t}$.

The following sections develop expressions for the transformation rate $\dot{v}_{t}$ and slip rate $\dot{\gamma}_{t}$ using a phase field formulation and crystal plasticity formulation, respectively.

\subsection{Phase Field Formulation}

The phase field method is an energy-based framework to predict the evolution of a set of field variables. The volume fraction $v_{t}$ of martensite CVs at a material point are chosen as the field variables. Following Artemev et al. (Artemev et al., 2001), the evolution of the order parameters is assumed to follow the Ginzburg-Landau kinetic equation.

$$
\dot{v}_{t}=\mathbf{M}_{t u} f_{u}^{\text {trans }}
$$

Here, the mobility matrix $\mathbf{M}_{t u}$ can in theory be a function of the field variables, temperature and strain. In this case, it is assumed to be a scalar constant. The driving force $f_{u}^{\text {trans }}$ for phase transformation is defined as the work conjugate of $v_{u}$ at a material point. The free energy function $\Psi_{R}$ for a unit volume mixture of austenite and martensite CVs is written in terms of contributions from the elastic strain energy, the chemical free energy and a gradient-based interface energy (Ammar et al., 2009; Anand, 2012; Jin et al., 2001; Wang and Khachaturyan, 1997), 
In Eq. 5, the elastic stiffness tensor $\mathbf{C}$ is calculated using the rule of mixtures of the austenite stiffness $\mathbf{C}_{\mathrm{A}}$ and the martensite stiffness $\mathbf{C}_{\mathrm{M}}$, so that $\mathbf{C}=v_{A} \mathbf{C}_{A}+v_{M} \mathbf{C}_{M}$. The elastic strain is calculated from the elastic deformation gradient, $\mathbf{E}^{e}=1 / 2\left(\mathbf{F}^{\mathbf{e T}} \mathbf{F}^{\mathbf{e}}-\mathbf{I}\right)$. The interface term consists of the spatial gradient $\nabla v_{u}$ of the order parameter and an interface energy coefficient $\mathbf{K}_{t u}$. The interface energy coefficient is assumed to be isotropic and independent of temperature, the field variables and the kinematic variables. Thus, it reduces to a scalar constant.

Assuming force balance, energy conservation and the $2^{\text {nd }}$ law of thermodynamics (Gurtin, 2000), the driving force per unit volume for phase transformation can be written as

$$
f_{t}^{\text {trans }}=\mathbf{F}^{\mathbf{e T}} \mathbf{F}^{\mathbf{e}} \mathbf{T}^{*} \mathbf{U}_{t}^{\text {trans }}-\frac{\partial \Psi_{R}}{\partial v_{t}}+\operatorname{div}\left(\frac{\partial \Psi_{R}}{\partial \nabla v_{t}}\right)=\mathbf{F}^{\mathbf{e T}} \mathbf{F}^{\mathbf{e}} \mathbf{T}^{*} \mathbf{U}_{t}^{\text {trans }}-\frac{\partial f_{L P}\left(v_{t}, \theta\right)}{\partial v_{t}}+\mathbf{K}_{\boldsymbol{t} \boldsymbol{u}} \nabla^{2} v_{u} \quad 6
$$

Thus, the driving force has contributions from the external stress, chemical free energy change between austenite and martensite, and an interface term. $\mathbf{T}^{*}$ is the $2^{\text {nd }}$ Piola-Kirchhoff stress, which is the work conjugate to the Green strain, $\mathbf{E}=1 / 2\left(\mathbf{F}^{\mathbf{T}} \mathbf{F}-\mathbf{I}\right)$. The chemical free energy $f_{L P}$ is defined in terms of a Landau-type polynomial (Artemev et al., 2001).

The chemical free energy relative to pure austenite is expressed in terms of a linear function of temperature and a sixth-order, Landau-type polynomial of the order parameter $v_{t}$,

$$
f_{\mathrm{LP}}\left(v_{t}, \theta\right)=\lambda\left[\begin{array}{c}
\left(\theta-\theta_{\text {trans }}\right)\left(p \sum_{i=1}^{N_{t}} v_{i}^{2}+q\left(\sum_{i=1}^{N_{t}} v_{i}^{2}\right)^{2}\right)+ \\
\left(b \sum_{i=1}^{N_{t}} v_{i}^{2}+d \sum_{i=1}^{N_{t}} v_{i}^{4}+f\left(\sum_{i=1}^{N_{t}} v_{i}^{2}\right)^{2}\right)
\end{array}\right]
$$

This form of the polynomial has been used extensively in the phase-field literature (Artemev et al., 2000; Gao et al., 2014; Wang and Khachaturyan, 1997). It has two characteristics. First, the minima at $v_{t}=0$ and $v_{t}=1$ correspond to the austenite and martensite phases respectively. Second, the polynomial provides activation barriers to the forward $(A \rightarrow M)$ and reverse $(M \rightarrow A)$ phase transformations. The activation barriers represent an energetic penalty incurred as the crystal structure transforms through unstable elastically-deformed intermediate configurations. An asymmetric activation barrier $\left(\Psi_{\text {activation }}^{A \rightarrow M} v s . \Psi_{\text {activation }}^{M \rightarrow A}\right)$ has been postulated by first-principles calculations (Kibey et al., 2009) and has been used extensively in the modeling of phase transformations (Chen and Schuh, 2015; Levitas and Preston, 2002). Figure 1 schematically shows the Landau polynomial at various temperatures.

Thus, Eqs. 4 and 6 provide the key expressions for the evolution of the field variables (martensite fractions). The next task is to develop an expression for the evolution in austenite slip activity. 


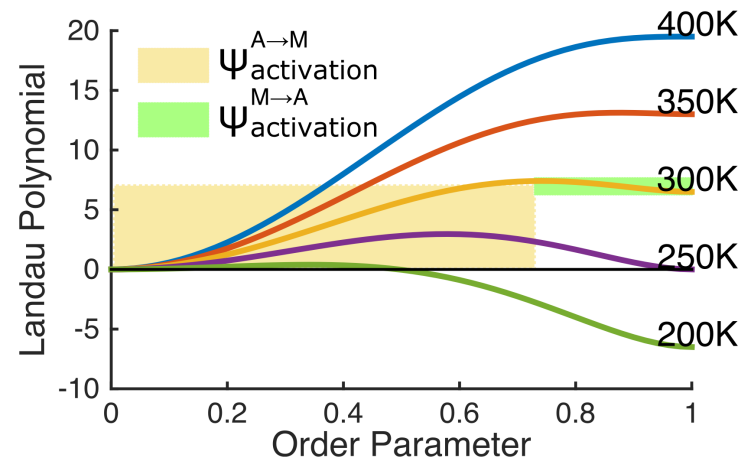

FIGURE 1 Form of the Landau polynomial at various temperatures. The austenite (A) and martensite (M) phases are in equilibrium at $250 \mathrm{~K}$. 


\subsection{Crystal Plasticity Law}

Slip is modeled in terms of the crystal plasticity formulation of Peirce et al. (Peirce et al., 1982). This formulation provides a rate dependent power-law type relation for slip evolution on different slip systems. A phenomenological hardening rule is expressed in terms of a set of self and latent hardening parameters. This formulation was proposed initially to study inhomogeneous deformation in metals and it has been used subsequently to model plastic deformation in a variety of metals and alloys (Kalidindi et al., 1992; Zambaldi et al., 2012). This continuum approach captures plasticity in terms of slip rate rather than the kinematics of individual dislocations. It is worth noting that the number of dislocations observed in the TEM-based experiments on NiTi single crystals is too large for discrete dislocation dynamics type of models (Bowers et al., 2014; Norfleet et al., 2009). Hence a continuum approach is suitable.

The slip rate $\dot{\gamma}_{s}$ on slip system $s$ is related to the resolved shear stress $\tau_{s}$ and a reference strength $g_{s}$ of a slip system.

$$
\begin{gathered}
\dot{\gamma}_{s}=\dot{\gamma}_{0}\left(\frac{\tau_{s}}{g_{s}}\right)^{\frac{1}{m}} \operatorname{sign}\left(\tau_{s}\right) \\
\tau_{s}=\mathrm{F}^{\mathrm{eT}} \mathrm{F}^{\mathrm{e}} \mathrm{T}^{*} \cdot \mathrm{S}_{t}^{\mathrm{slip}}
\end{gathered}
$$

The reference strength evolves as

$$
\dot{g}_{s}=\sum_{r=1}^{N_{s}} h_{r}\left(Q+(1-Q) \delta_{s r}\right)\left|\dot{\gamma}_{r}\right| \quad \text { and } \quad h_{r}=h_{0}\left(1-\frac{g_{r}}{g_{\text {sat }}}\right)^{a}
$$

Here $\dot{\gamma}_{0}$ is a reference slip rate, $m$ is the strain rate exponent, $Q$ is the ratio of self to latent hardening, $h_{0}$ is the self hardening coefficient, $g_{\text {sat }}$ is the saturation strength of a slip system, and $a$ is the hardening exponent.

Slip evolution obtained from Eqs. 8 and 10, together with the martensite evolution equation, provide the details needed to calculate inelastic deformation gradient in Eq. 3. The coupling between phase transformation and plasticity is mediated through the local stress $\mathbf{T}^{*}$. The constitutive relations are numerically solved in a finite element framework.

\subsection{Finite Element Implementation}

The aim of the numerical procedure is to obtain the Cauchy stress ( $T(\tau)$ ), inelastic deformation gradient $\left(\mathrm{F}^{\text {inel }}(\boldsymbol{\tau})\right)$, martensite $\mathrm{CV}$ volume fraction $\left(\nu_{m}(\tau)\right)$ for transformation system $m$, slip activity $\left(\gamma_{s}(\tau)\right)$ on slip system $s$, and slip system hardness $\left(g_{s}(\tau)\right)$ at the current time increment $\tau=t+\Delta t$. These are computed in terms of the proposed total deformation gradient $(\mathrm{F}(\tau))$ and temperature $(\theta(\tau))$ plus the values of all quantities at the prior time $t$. This is achieved through a six-step explicit numerical integration approach.

First, a trial value of the inelastic deformation gradient is assumed to be equal to that in the previous time-increment $\left(\mathbf{F}^{\text {inel(trial) }}(\tau)=\mathbf{F}^{\text {inel }}(t)\right)$. Eq. 1 then furnishes a trial elastic deformation gradient $\left(F^{\mathrm{e}(\text { trial })}(\tau)\right)$. Second, the elastic deformation gradient is used to compute a trial Green's strain and $2^{\text {nd }}$ Piola-Kirchhoff stress. 


$$
\begin{gathered}
\mathbf{E}^{\mathrm{e}^{(\text {trial })}(\tau)}=\frac{1}{2}\left(\mathbf{F}^{\mathrm{e}^{(\text {trial })}(\tau)}(\tau) \mathbf{F}^{\left.\mathrm{e}^{(\text {trial })}(\tau)-\mathbf{I}\right)}\right. \\
\mathbf{T}^{*(\text { trial })}(\tau)=\mathbf{C}\left(\mathbf{E}^{\mathrm{e}(\text { trial })}(\tau)-\mathbf{A}^{\text {therm }} \Delta \theta\right)
\end{gathered}
$$

The thermal expansion coefficient $\left(\mathbf{A}^{\text {therm }}\right)$ and stiffness matrix $\mathbf{C}$ are calculated using a rule-ofmixtures approach $\left(\mathbf{C}=v_{A} \mathbf{C}_{A}+v_{M} \mathbf{C}_{M}\right)$. Third, the resolved stresses are calculated on the transformation and slip systems.

$$
\begin{gathered}
\tau_{s}^{\text {slip }}(\tau)=\mathbf{F}^{e T(\text { trial })}(\tau) \mathbf{F}^{e(\text { trial })}(\tau) \mathbf{T}^{*(\text { trial })}(\tau) \cdot \mathbf{S}_{s}^{\text {slip }} \\
\tau_{m}^{\text {trans }}(\tau)=\mathbf{F}^{e T(\text { trial })}(\tau) \mathbf{F}^{e(\text { trial })}(\tau) \mathbf{T}^{*(\text { trial })}(\tau) \cdot \mathbf{U}_{m}^{\text {trans }}
\end{gathered}
$$

The fourth step uses these quantities to compute the evolution in both the volume fractions of martensite CVs (Eqs. 4 and 6) and the activities on each of the austenite slip systems (Eq. 8). Fifth, an updated inelastic deformation gradient $\left(\mathrm{F}^{\text {inel }}(\tau)\right)$ is calculated.

$$
\mathbf{F}^{\text {inel }}(\boldsymbol{\tau})=\left(\mathbf{1}+\sum_{m=1}^{N_{t}} \dot{v_{m}}(\tau) \mathbf{U}_{m}^{\text {trans }}+v_{A} \sum_{t=1}^{N_{s}} \dot{\gamma_{t}}(\tau) \mathbf{S}_{t}^{\text {slip }}\right) \mathbf{F}^{\text {inel(trial) }}(\boldsymbol{\tau})
$$

Sixth, the updated inelastic deformation gradient is used to compute an updated elastic deformation gradient (Eq. 1), elastic strain (Eq. 11) and stress (Eq. 12). The Cauchy stress is therefore calculated as

$$
\mathbf{T}(\boldsymbol{\tau})=\frac{1}{\operatorname{det}\left(\mathbf{F}^{e}(\boldsymbol{\tau})\right)} \mathbf{F}^{e}(\boldsymbol{\tau}) \mathbf{T}^{*}(\tau) \mathbf{F}^{e T}(\boldsymbol{\tau})
$$

The numerical scheme is implemented as a user material subroutine (VUMAT) in the Abaqus commercial FEM framework (ABAQUS, 2008). A key advantage of the FEM implementation over other methods (e.g., Fourier based techniques) is the relative ease of modeling complex geometries and boundary conditions and the ability to incorporate material rotation and stretching due to finite deformations. An explicit numerical scheme provides two benefits. First, implementing a Jacobian $(\partial \mathbf{T} / \partial \mathbf{E}$ and $\partial \mathbf{T} / \partial \theta)$ is not necessary, simplifying the model development. Second, the ABAQUS FEM package provides efficient domain decomposition, allowing parallel execution of simulations with $\sim 700,000$ degrees-of-freedom in $\sim 12$ hours on 64 processors. A disadvantage of the explicit approach is the conditional stability, which requires a small time increment $(\Delta t)$. Also, the explicit scheme solves a dynamical problem, $\ddot{u}^{(i)}=\mathbf{M}^{-1}\left(\mathrm{~F}_{\text {applied }}^{(i)}-\mathrm{F}_{\text {internal }}^{(i)}\right)$, where $\ddot{u}^{(i)}$ is the acceleration of the $i^{\text {th }}$ node, $\mathbf{M}$ is the lumped mass matrix, $\mathbf{F}_{\text {applied }}$ is the applied load and $\mathbf{F}_{\text {internal }}$ is the internal force. A consequence of this is the inclusion of inertial effects on the microstructure evolution. Mass scaling (scale factor $\approx 10^{6}$ ), which is typically used to make the stable time increment $(\Delta t)$ larger, also increases the inertia of the system. Hence, the inertial effects can be significant based on the loading rate and the density. The aim of this work is to model a quasi-static response of the material and thus inertial effects are undesirable. To mitigate these effects, a viscous drag force $\left(f_{\mathrm{v}}=-c_{\mathrm{vb}}(v-\right.$ $\left.v_{\text {ref }}\right) V_{\text {elem }}$ ) is applied at each material point (ABAQUS, 2008). Here $c_{\mathrm{vb}}$ is a constant ( $=35 \mathrm{Ns} / \mathrm{m}^{4}$ ) determined by trial-and-error, $v$ is the element velocity and $V_{\text {elem }}$ is the volume of the element. $v_{\text {ref }}$ is set to zero. The addition of viscous drag is seen to stabilize the simulations.

The development of the constitutive law assumes that the representative volume element or the material point is a single crystal. However, the numerical implementation rotates the stiffness tensor 
(C), transformation stretch matrices ( $\mathbf{U}^{\text {trans }}$ ), and Schmid tensor for slip ( $\mathbf{S}^{\text {slip }}$ ) from a crystal to a global coordinate system. Hence, this framework can be used to model polycrystals if the local crystallographic orientation is provided at the material points.

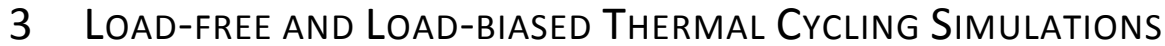

Thermal cycling simulations are carried out using a cylindrical model created with $1.14 \times 10^{5}$ hexahedral, reduced-integration elements (C3D8RT in ABAQUS FEM suite). A reduced-integration element is more computationally efficient than the full integration counterpart because kinematical calculations are performed at $1 / 8^{\text {th }}$ the number of integration points. Figure 2 shows the model geometry and boundary conditions. All nodes on the base of the cylinder are constrained to stay in the $Y=0$ plane $\left(u_{Y}(Y=0)=0\right)$ but they can slide freely in the plane (traction $\left.T_{X}=T_{Z}=0\right)$. The node at the center of the base is fully constrained $(\boldsymbol{u}(X=Y=Z=0)=0)$. The boundary conditions on the base of the cylinder are imposed to avoid rigid body motion. For the load-biased simulations described below, the top surface is not traction-free but all other surfaces are traction free. As shall be seen, martensite laths in these simulations are $\sim 4$ elements thick and such laths are $\sim 20 \mathrm{~nm}$ thick based on TEM observations (e.g., Norfleet et al. 2009; Bowers et al. 2014). Thus, each element $\sim 5 \mathrm{~nm}$ in size and the overall model is $\sim 300 \mathrm{~nm}$ wide and $150 \mathrm{~nm}$ tall. Due to limited computational resources, it is difficult to address polycrystalline aggregates of SMA.

Two single-crystal load-free thermal cycle simulations are carried out. In the first simulation, plasticity is turned off by setting the reference strength $\left(g_{s}\right)$ to a very large value ( $\left.10^{6} \mathrm{MPa}\right)$. This is referred to as the without-plasticity case. In the second simulation, the reference strength is set to $160 \mathrm{MPa}$. This is referred to as the with-plasticity case. The reference strength value is chosen such that the critical stresses for plasticity and phase transformation are comparable. This was determined by performing a sequence of trial simulations. In each case, two thermal cycles are imposed.

Two single-crystal load-biased thermal cycling simulations are also carried out. In each case, a bias stress of -60 MPa is applied in the $Y$ direction on the top surface of the cylinder. These two simulations are identical to the with-plasticity and without-plasticity cases described for the load-free thermal cycling simulations except that the constant biasing stress is applied. In this case, large localized plastic deformation occurs in the second cycle of the load-biased simulations. Additionally, the step size in the explicit FE solver (ABAQUS) cannot be adaptively reduced to repeat the calculation at that time step. This leads to element distortion in the second cycle of the load-biased simulations, causing the simulation to abort. Hence only the first cycle is carried out. Using a very small time step throughout the load-biased simulation can mitigate this effect. However that is computationally expensive.

During thermal cycling, the temperature is varied from $400 \mathrm{~K}$ to $150 \mathrm{~K}$ at a rate of $16.7 \mathrm{~K} / \mathrm{s}$, thus passing through the austenite-martensite equilibrium temperature $\left(\theta_{\text {trans }}=250 \mathrm{~K}\right)$. All elements in the FE mesh are prescribed to have the same specified temperature at a given time increment. This automatically satisfies thermal equilibrium. At the start of the first thermal cycle, inhomogeneous nucleation is simulated by specifying a non-zero order parameter $\left(v_{2}^{N 1}=0.6\right.$ and $\left.v_{1}^{N 2}=0.4\right)$ at two elements marked as $\mathrm{N}_{1}$ and $\mathrm{N}_{2}$ in Figure 2. The presence of inhomogeneous sites is inferred from fluctuations in chemistry and stress in physical experiments as well as thermal fluctuations in molecular dynamics simulations (Liu et al., 2014; Morrison et al., 2015). A single crystal specimen is studied by assigning the same crystallographic orientation to all elements of the mesh. The crystal- 
to-global correspondence is: [ $\left[\begin{array}{lll}1 & 0 & 0\end{array}\right]\left\|\mathrm{X},\left[\begin{array}{lll}0 & 1 & 0\end{array}\right]\right\| \mathrm{Y}$ and [ $\left[\begin{array}{lll}0 & 0 & 1\end{array}\right] \| \mathrm{Z}$. The computational efficiency was increased by setting the interface energy term $\mathbf{K}_{t u}$ in Eq. 6 to zero. This eliminates the need to calculate the gradient of the order parameter over an irregular mesh and reduces the computational expense by approximately $50 \%$. Despite setting the interface term to zero, there is still an energetic penalty at the interfaces. This is due to the local stresses introduced by the discrete nature of the finite element mesh. Because of this energy penalty, we obtain martensite laths which are multiple elements wide (e.g. Figure 6). This differs from spectral methods, which require a non-zero gradient term in order to obtain martensite laths which span multiple grid points.

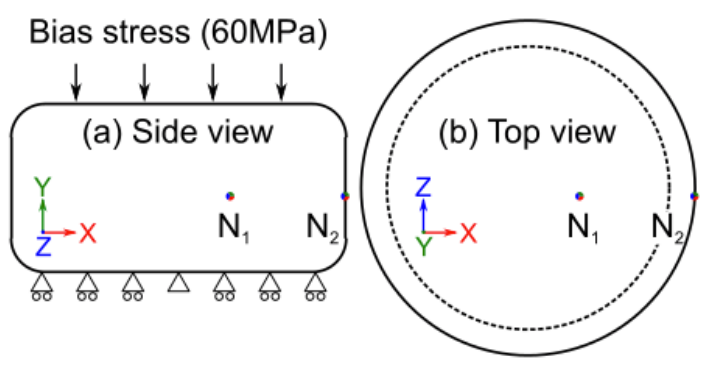

FIGURE 2 Geometry of the models created for thermal cycling simulations. The bias stress in (a) is applied in the case of load-biased thermal cycling simulations. 
TABLE 1 Cubic to tetragonal transformation strain matrices for the three CVs. Below each matrix, a graphical representation of the deformation induced is shown.

$E_{1}=\left(\begin{array}{ccc}-0.03 & 0 & 0 \\ 0 & -0.03 & 0 \\ 0 & 0 & 0.06\end{array}\right) \quad E_{2}=\left(\begin{array}{ccc}-0.03 & 0 & 0 \\ 0 & 0.06 & 0 \\ 0 & 0 & -0.03\end{array}\right) \quad E_{3}=\left(\begin{array}{ccc}0.06 & 0 & 0 \\ 0 & -0.03 & 0 \\ 0 & 0 & -0.03\end{array}\right)$

TABLE 2 Cubic slip systems modeled in the austenite phase.

\begin{tabular}{|c|c|c|}
\hline Slip system & Slip plane & Burgers vectors \\
\hline 1 & $\left(\begin{array}{lll}0 & 1 & 1\end{array}\right)$ & {$\left[\begin{array}{lll}1 & 0 & 0\end{array}\right]$} \\
\hline 2 & $\left(\begin{array}{lll}0 & 1 & \overline{1}\end{array}\right)$ & {$\left[\begin{array}{lll}1 & 0 & 0\end{array}\right]$} \\
\hline 3 & $\left(\begin{array}{lll}1 & 0 & 1\end{array}\right)$ & {$\left[\begin{array}{lll}0 & 1 & 0\end{array}\right]$} \\
\hline 4 & $\left(\begin{array}{lll}1 & 0 & \overline{1}\end{array}\right)$ & {$\left[\begin{array}{lll}0 & 1 & 0\end{array}\right]$} \\
\hline 5 & $\left(\begin{array}{lll}1 & 1 & 0\end{array}\right)$ & {$\left[\begin{array}{lll}0 & 0 & 1\end{array}\right]$} \\
\hline 6 & $(\overline{1} 10)$ & {$\left[\begin{array}{lll}0 & 0 & 1\end{array}\right]$} \\
\hline
\end{tabular}

A model cubic-to-tetragonal transformation system is used. This type of transformation is observed in Fe-30at.\%Pd (Cui et al., 2004), Ni-36at.\%Al (Martynov et al., 1983) and Fe-24\%Pt (Tadaki and Shimizu, 1975) alloys. Table 1 shows the transformation stretch matrices and the deformed shape of a cube after imposing each of the three energetically equivalent transformations. The transformation stretches $\boldsymbol{E}_{\mathrm{t}}$ are such that their middle eigenvalue is not equal to 1 . Hence a single CV cannot form a stress-free interface with austenite (Ball and James, 1987; Bhattacharya, 2003). However, a twinned martensite region can form such an interface. Calculations based on the crystallographic theory of martensite (CTM) reveal that there are six possible $M-M$ interfaces and twenty-four A-M interfaces (Bhattacharya, 2003). In terms of the order parameter $\left(v_{t}\right)$, an A-M interface separates a region with $v_{t}=0$ from that with $v_{t}=1$. A M-M interface separates two regions with $v_{t}=1$ and $v_{u}=1$ such that $t \neq$ $u$.

Six austenite slip systems with $\left\{\begin{array}{lll}1 & 1 & 0\end{array}\right\}$ type slip planes are modeled. The slip plane normal and Burgers vector for the slip systems are listed in Table 2. The crystallographic orientation of the

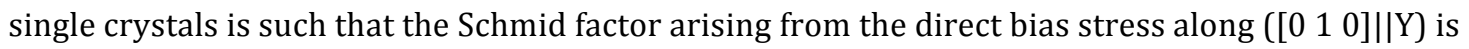
zero for all six slip systems. All other simulation parameters and constants in the constitutive relations are listed in Table 3. A relatively large strain rate exponent $(m=0.5)$ for plasticity was chosen because simulations employing smaller values (e.g., $m=0.02)$ characteristic of macroscopic tension tests on FCC metals were unstable due to rapid onset of slip. A potential consequence of a large $m$ is an increased rate dependence in austenite slip activity. The larger $m$ is expected to produce smaller values of plastic strain in regions that undergo a relatively large deformation rate. 
The model tetragonal transformation system is chosen to systematically study the interaction between phase transformation microstructure and austenite slip. The qualitative results reported here are general and expected to hold for commonly used SMAs like NiTi as well as the model tetragonal transformation system. The transformation strain modeled in this case (approx. 6\%) is comparable to equiatomic NiTi.

A set of simulations was carried out to assess the sensitivity of the results to the mesh size and the effect of not incorporating the interface term $\left(\mathbf{K}_{t u}\right)$. To assess the effect of mesh density, step-quench simulations with the number of elements ranging between 50,000 and 2,000,000 were performed. The results did not show mesh dependence for meshes with more than $\sim 100,000$ hexahedral or $\sim 500,000$ tetrahedral elements. To assess the effect of the interface energy term, two simulations were carried out with $\mathbf{K}_{\mathrm{tu}}=0$ and $\mathbf{K}_{\mathrm{tu}}=1 \times 10^{-3} \mathrm{~J} / \mathrm{m}$. For these simulations, an identical mesh and the same step-quench temperature history was imposed. The martensite microstructure length scale is comparable in both simulations. The thermal cycling simulations were carried out after verifying that the chosen mesh $\left(1.14 \times 10^{5}\right.$ hex elements) is appropriate and the effect of a zero interface term is small for these simulations. The results are summarized in the next section.

TABLE 3 Material properties used in the thermal cycling simulations.

\begin{tabular}{ll}
\hline Elastic and thermal & Elastic constants $(\mathrm{GPa}): C_{11(\mathrm{~A})}=130 ; C_{22(\mathrm{~A})}=98 ; C_{44(\mathrm{~A})}=21 ; C_{\mathrm{M}}=C_{\mathrm{A}} / 2$ \\
& Thermal expansion coefficients $\left(10^{-6} / \mathrm{K}\right): \alpha_{\mathrm{A}}=11 ; \alpha_{\mathrm{M}}=6.6$ \\
\hline Phase transformation & Equilibrium transformation temperature $(\mathrm{K}): \theta_{\mathrm{T}}=250$ \\
& Latent heat of transformation/unit volume $(\mathrm{MPa}): \lambda_{\mathrm{T}}=20$ \\
& Landau polynomial coefficients: $p=1.0, q=-0.5, b=1.0, d=-2.0, \mathrm{f}=1.0$ \\
& Mobility $(/ \mathrm{s}): M=3.5 \times 10^{-2}$ \\
& Number of martensite CVs: $N_{\mathrm{T}}=3$ \\
& Interface energy term $(\mathrm{J} / \mathrm{m}), K_{t u}=0$ \\
\hline Austenite plasticity & Reference shear strain rate $(/ \mathrm{s}): \dot{\gamma}_{0}=0.02$ \\
& Strain rate exponent: $\mathrm{m}=0.5$ \\
& Initial slip system shear strength $(\mathrm{MPa}): g_{s}^{0}=160$ for the with-plasticity \\
& case and $g_{s}^{0}=10^{6}$ for the without-plasticity case. \\
& Self-hardening coefficient $(\mathrm{MPa}): h_{0}=1250$ \\
& Ratio of self to latent hardening: $\mathrm{Q}=1.4$ \\
& Hardening exponent: a $=0.1$ \\
& Saturation shear strength $(\mathrm{MPa}): g_{\mathrm{sat}}=1000$ \\
& Number of slip systems: $N_{\mathrm{S}}=6$
\end{tabular}

\section{Results}

\subsection{Load-free Thermal Cycling}

Figures 3a and $\mathbf{3 b}$ show the macro martensite fraction $\left(v_{M}=v_{1}+v_{2}+v_{3}\right)$ vs. temperature $(\theta)$ for the without-plasticity and with-plasticity cases, respectively. During first cycle, the transformation begins at the nucleation sites and for both cases, only $60 \%$ of the sample transforms to martensite by the time the minimum temperature $(\theta=150 \mathrm{~K})$ is reached (points $\mathrm{A}$ and $\mathrm{A}^{\prime}$, respectively). This highlights the kinetic nature of the phase transformation (Eq. 4) during rapid cooling (16.7K/s). Upon heating, both samples fully transform to martensite by the time $\theta=165 \mathrm{~K}$ is reached (points $\mathrm{A}^{*}$ and $A^{* *}$, respectively). Above this temperature to $\sim 240 \mathrm{~K}$ (e.g., between points $\mathrm{A}^{*}$ and $\mathrm{B}$ ), the martensite fraction remains constant. With continued heating to $400 \mathrm{~K}, v_{\mathrm{M}}$ decreases precipitously 
(e.g., between points B and P) and then decreases at a more modest rate with small fluctuations that gradually reduce (i.e., between points $\mathrm{P}$ and $\mathrm{Q}$ ). At the end of the first cycle, 3.8\% martensite does not transform back in the without-plasticity case vs. $2.4 \%$ in the with-plasticity case. The incomplete transformation could be a manifestation of the choice of the mobility $(M)$ value (Eq. 4, Table 3). However, this is not expected to alter the fundamental trends in martensite evolution during the first cycle.

During second cycle, the $\mathrm{A} \rightarrow \mathrm{M}$ transformation begins earlier (i.e., at a higher temperature) compared to the first cycle. Also, the $\mathrm{A} \rightarrow \mathrm{M}$ transformation reaches completion by the time the minimum temperature $(\theta=150 \mathrm{~K})$ is reached (points $C$ and $C^{\prime}$, respectively). This is a potential consequence of the accumulated slip and residual martensite present at the end of the first cycle. In the withoutplasticity case (Figure 3a), the reverse transformation $(\mathrm{M} \rightarrow \mathrm{A})$ path is similar for the two cycles. In the with-plasticity case (Figure $3 \mathrm{~b}$ ), the reverse transformation path in the second cycle is slightly shifted to higher temperature compared to the first cycle.

Figures 3c and 3d show the evolution in individual martensite CVs vs. temperature for the withoutplasticity and with-plasticity cases, respectively. In both cases, the individual CVs grow equally in volume fraction to pt. A and they are comparable upon initial heating to points $\mathrm{A}^{*}$ and $\mathrm{A}^{* *}$, respectively. Upon continued heating to points $\mathrm{B}$ and $\mathrm{B}^{\prime}$, respectively, the samples are fully martensitic and CV1 increases in volume fraction while CV2 and CV3 either decrease or hold constant. Thus, M-M interfaces displace during this portion. Above this point, CV1 continues to increase even though the overall martensite volume fraction begins to decrease. Above $\sim 260 \mathrm{~K}$, all volume fractions decrease and above $300 \mathrm{~K}$, oscillations in volume fraction arise from the dynamical nature, which includes the propagation of stress waves through the sample - a consequence of the mass scaling.

During the second cycle, the $\mathrm{A} \rightarrow \mathrm{M}$ transformation is equal among the three CVs down to pt. A (160 $\mathrm{K})$ but the amount transformed is about twice that in the first cycle. Upon continued cooling to 150 $\mathrm{K}$, the volume fraction of CV1 increases above the others. Upon heating, CV1 continues to dominate and it reaches an even greater volume fraction than in the first cycle. At the end of the second heating cycle, fluctuations are observed as in the first cycle. Overall, the trends in the without-plasticity and with-plasticity cases are comparable during cooling in the first and second cycles. The dominance of CV1 is predicted to increase with continued cycling (cycle 2 vs. cycle1) and is modestly smaller in the with-plasticity case. The with-plasticity case also has a relative increase in the volume fraction of CV2 and a decrease in CV3. In both cases, the relative ordering of the CV volume fractions is a consequence of the boundary conditions and the bias in the inhomogeneous nucleation sites at points $N_{1}$ and $N_{2}$ in Figure 2. The boundary conditions do not favor CV2 since it produces a large distortion in the $Y$ direction, which is restricted on the bottom face of the model. The nucleation sites have the initial conditions $v_{2}^{N 1}=0.6$ and $v_{1}^{N 2}=0.4$, which do not favor CV3. If the initial conditions are changed to $v_{2}^{N 1}=0.2$ and $v_{3}^{N 2}=0.6$, comparable volume fractions of CV1 and CV3 form and CV2 is relatively smaller. 
(a)

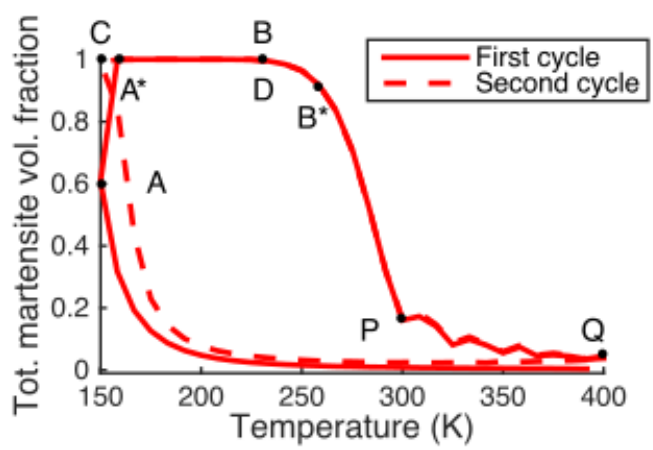

(c)

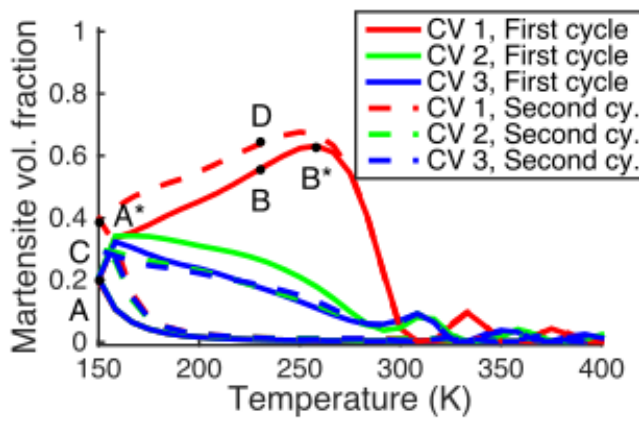

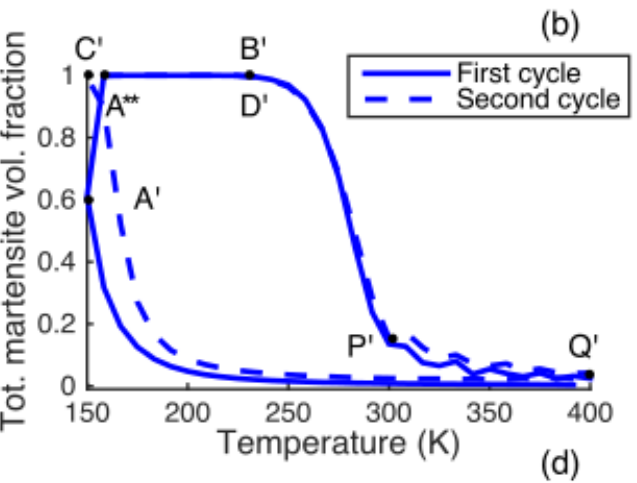

(d)

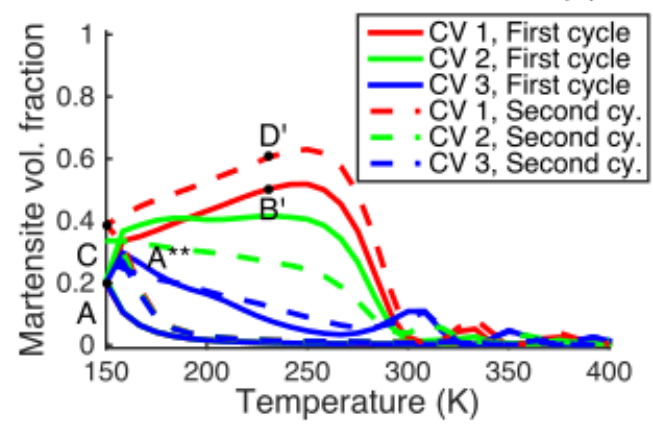

FIGURE $3(a, b)$ Total martensite volume fraction as a function of temperature for load-free thermal cycling. (a) Without-plasticity case and (b) with-plasticity case. (c, d) Volume fractions of the individual CVs as a function of temperature for load-free thermal cycling. (c) without-plasticity case, (d) with-plasticity case. 
The average equivalent plastic strain at the end of two cycles is small $(0.2 \%)$ compared to the strain produced by phase transformation (6\%). The rate of plastic strain with temperature (i.e., slope) is largest at the end of the heating (M-A) cycle and at the start of the cooling (A-M) cycle. There are also significant increments in plastic strain at the end of the cooling (A-M) cycle and start of the heating (M-A) cycle. In the latter cases, the volume fraction of the plastically-deforming phase (austenite) is small yet the increment in average plastic strain is significant. This suggests profuse amounts of plasticity in the last regions of austenite to transform to martensite and in the first regions of austenite to nucleate curing heating. The plastic strain increase during cooling is predicted to be larger in the second cycle $(0.1 \%)$ vs. the first cycle $(0.02 \%)$. This occurs despite the hardening in the crystal plasticity law (Eq. 10 and Table 3). Conversely, the plastic strain increase during heating is predicted to be larger in the first cycle $(0.06 \%)$ vs. the second cycle $(0.02 \%)$.

\subsection{Load-biased Thermal Cycling}

Figures $5 \mathbf{a}$ and $\mathbf{5 b}$ show the macro martensite volume fraction $\left(v_{M}\right)$ vs. temperature $(\theta)$ for the loadbiased simulations, where a bias stress of $-60 \mathrm{MPa}$ is applied along the $\mathrm{Y}$ axis. The forward transformation starts earlier $\left(\theta_{\mathrm{MS}}=222 \mathrm{~K}\right)$ compared to the load-free case $\left(\theta_{\mathrm{MS}}=162 \mathrm{~K}\right)$ and unlike the load-free case, it completes when the minimum temperature $(150 \mathrm{~K})$ is reached. The responses for the with-plasticity (Figure 5(a)) and without-plasticity cases (Figure 5(b)) are identical.

Figures $\mathbf{5 c}$ and $\mathbf{5 d}$ show the evolution in volume fractions of the individual CVs. CV2 is essentially eliminated by the imposed compressive stress. This can be rationalized on the basis of the transformation strain generated by the three CVs. The applied compressive direct stress in the $Y$ direction favors CV1 and CV3 since they produce 3\% compression in the Y direction, whereas CV2 is not favored since it produces $6 \%$ extension in the $Y$ direction. Similar to the load-free cases, the initial conditions on the nucleation sites cause the volume fractions of CV1 and CV3 to diverge during cooling (pt. E*, Figure 5c) and CV1 to dominate during the heating part of the thermal cycle.

Figure $4 \mathbf{b}$ shows the average equivalent plastic strain (epls(eq) $v s$. martensite fraction $\left(v_{M}\right)$ for the load-biased case. Three observations are made. First, the load-biased case accumulates more plastic strain $(0.1 \%)$ at the end of the first cycle compared to the load-free case $(0.08 \%)$ (compare points $Q$ and S). Second, more plastic strain is generated during the heating part ( $0.065 \%$ at point $S$ ) compared to the cooling part $(0.035 \%$ at point $\mathrm{E})$. Third, the rate of plastic strain accumulation is greater toward the end of the cycle (points $\mathrm{R}$ to $\mathrm{S}$ ).

These results provide a macro scale overview of the features of phase fractions and slip evolution. The next section presents a detailed discussion of the micro-scale features. 
(a)

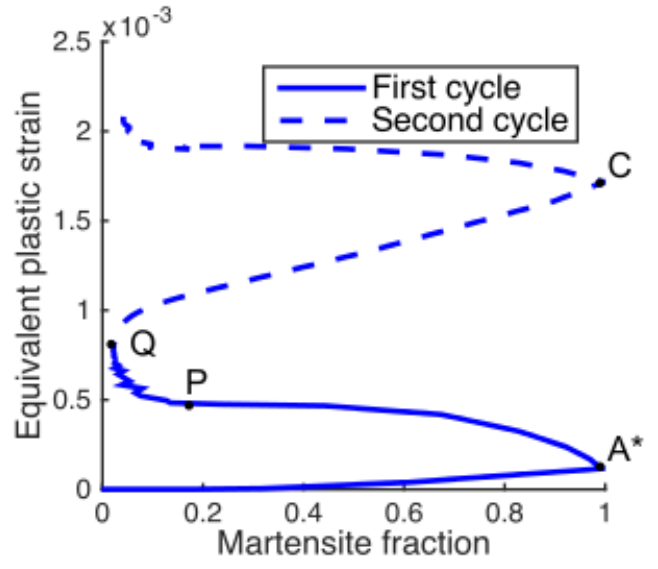

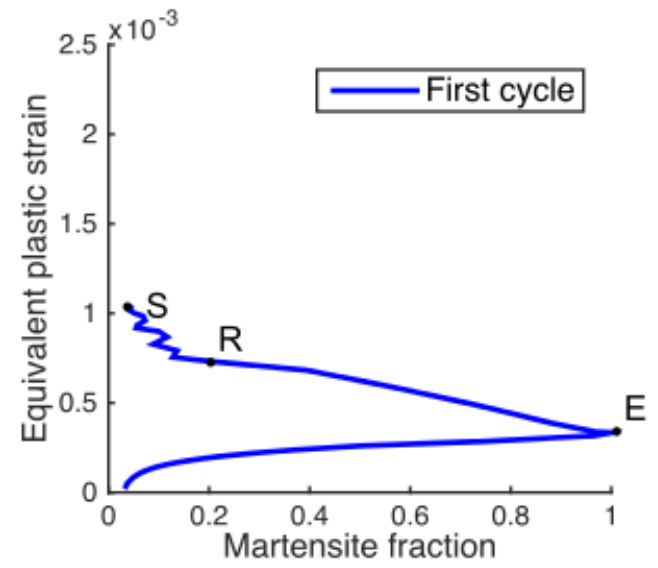

FIGURE 4 Plot of the average equivalent plastic strain vs. the volume-averaged total martensite volume fraction, (a) load-free thermal cycling and (b) load-biased thermal cycling. 
(c)
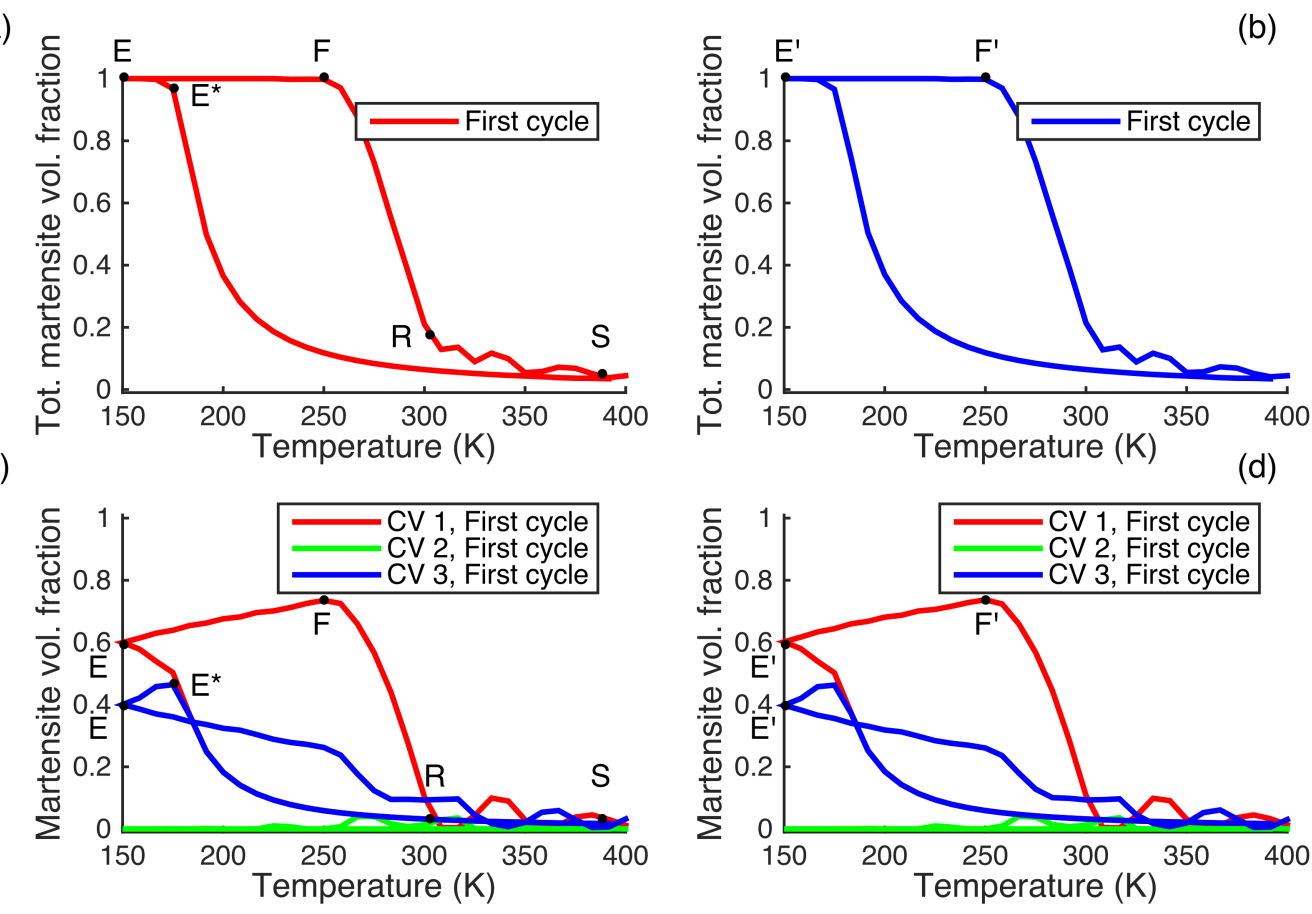

Figure $5(\mathrm{a}, \mathrm{b})$ Total martensite volume fraction as a function of temperature for load-biased thermal cycling. (a) Without-plasticity case, (b) with-plasticity case. (c, d) Volume fractions of the individual CVs as a function of temperature for load-biased thermal cycling. (c) without-plasticity case, (d) withplasticity case. 


\section{Discussion}

The results of the thermal cycling simulations can be used to explore the relation between martensite microstructure and austenite slip system activation and the effect of plasticity on measurable parameters such as the transformation temperatures. Three key questions are answered. First, where does plasticity occur in austenite with respect to the evolving martensite microstructure? This question relates to the spatial distributions of stress arising from incompatibility between austenite and martensite. Second, when does plasticity occur? The stress field generated by A-M interfaces is likely to be different in the first vs. second cycles and heating vs. cooling portions. This is expected to affect the rate of plasticity generation. Third, what are the effects of austenite slip on subsequent phase transformation? This question explores the effect of stress relaxation due to slip on measurable parameters such as transformation temperature and the spatial features of the martensite microstructure. This section also explores the relevant features of the martensite microstructure.

\subsection{Spatial Features of Martensite and Slip in Austenite}

Low energy interfaces between $\mathrm{A}-\mathrm{M}$ and $\mathrm{M}-\mathrm{M}$ is an output rather than an input of the model. The interface orientations predicted in the thermal cycling simulations match the predictions of the crystallographic theory of martensite (CTM)(Bhattacharya, 2003). The M-M interfaces are predicted by the CTM to be of the type $\left\{\begin{array}{lll}1 & 1 & 0\end{array}\right\}$. Figure $6\left(\right.$ a) shows the martensite microstructure at point $A^{*}$ of the load-free thermal cycle. M-M interfaces marked (1) have interface planes $\| \sim\left(\begin{array}{lll}0 & 1 & -1\end{array}\right)$. Those marked (4) have interface planes $\| \sim\left(\begin{array}{lll}1 & 0 & -1\end{array}\right)$. For the load-biased simulations, only CV1 and CV3 are present. They form interfaces of $\sim\left(\begin{array}{l}1 \\ 0\end{array} \pm 1\right)$ type. These are marked as (6) in Figure 8(e). In some instances, the observed orientation of the interface plane deviates from the CTM prediction. One such instance is marked as (7) in Figure 8(e). These deviations can be rationalized in two ways. First, formation of an interface plane with the theoretical orientation may result in a stressed state in the mesh, which can be relaxed by rotating the interface to a different orientation. Second, the CTM calculation assumes a stress-free state. In principle, plastic deformation can induce stress and therefore deviations from the CTM predictions.

Regions of plastic strain generated in the first cycle follow bands with a morphology similar to the martensite bands formed during the first cycle. Figure 6(a) shows the martensite microstructure formed at $166 \mathrm{~K}$ (point $\mathrm{A}^{* *}$ in Figure 3(b)) for the with-plasticity, load-free case. The CVs form straight interfaces marked as (1). Figure 6(b) shows the equivalent plastic strain (eplas(eq)) distribution at the end of first cycle (point $Q^{\prime}$ in Figure 3(b)). The plastic deformation is predominantly along thin bands marked as (2). These bands follow the boundaries between M-M interfaces formed during the first cycle. More M-M interfaces correspond to larger slip activity, marked as (4) and (5). This suggests that the stress field associated with accommodating impinging CVs is one of the factors responsible for slip in austenite. To quantify this observation, a partitioning scheme is devised to characterize each region of the model based on the local martensite microstructure. 


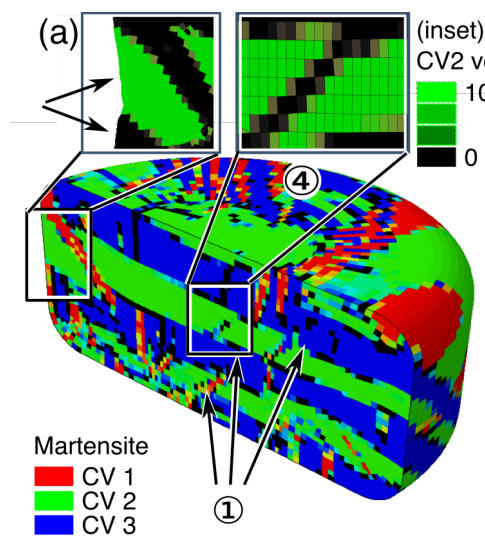

(b) $00 \%$

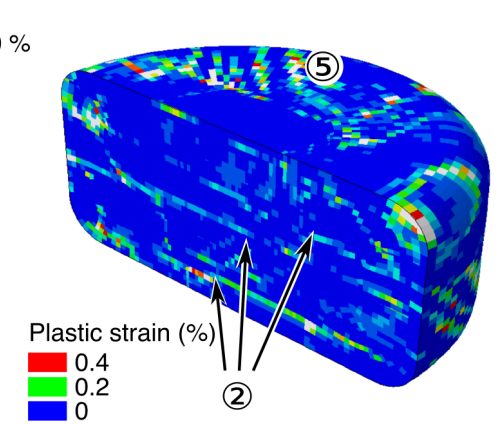

(c)

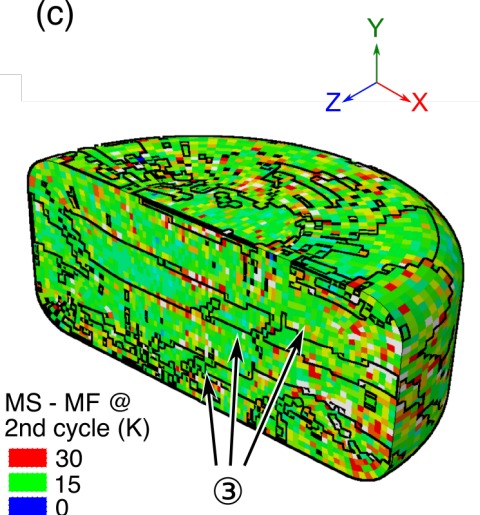

FIGURE 6 Spatial correlation between (a) the martensite microstructure formed during the first cycle of load-free thermal cycling. The left inset shows undulations at the sample edge (see arrows) due to the incorporation of large deformations/rotations in the model formulation. The deformation is scaled 5x. The right inset shows diffuse interfaces that are approximately 2-elements wide, (b) the plastic strain (eplas(eq) $)$ distribution at the end of the first cycle and (c) the difference in the MS and MF temperatures during the second cycle. Key features are marked in circles and referred to in the text. 
Based on the qualitative observation above, it is hypothesized that the plastic strain generated in a neighborhood is proportional to the density of incompatible interfaces formed. The density of incompatible interfaces is expressed in terms of the number of neighboring elements in which the dominant CV is different than that in the parent element. Figure $\mathbf{7}$ shows the results of the analysis, in which the plastic strain in parent elements is plotted vs. the number of dissimilar neighbors, based on the martensite microstructure formed during the first cycle. A numerical measure for the dissimilarity of the neighborhood is defined and the quantities in Figure 7 are calculated using a three-step approach. First, a martensite microstructure type $\left(M^{\text {elem }}\right)$ is assigned to each element based on the dominant $\mathrm{CV}$ formed at $166 \mathrm{~K}$ of the first cycle in the load-free thermal cycling simulation. The microstructure is fully martensitic at this point.

$$
M^{\text {elem }}= \begin{cases}1, & \text { if } v_{1}>60 \% \\ 2, & \text { if } v_{2}>60 \% \\ 3, & \text { if } v_{3}>60 \% \\ 4, & \text { else }\end{cases}
$$

Second, the number of neighbors with dissimilar $M$ elem is counted for each element. A 26-element neighborhood of each element is considered. This gives the quantity ( $N$ dissimilar) on the $\mathrm{X}$ axis of Figure 7. Third, the average equivalent plastic strain over all elements with the same $N$ dissimilar is calculated. This gives the quantity on the $\mathrm{Y}$ axis.

Plastic strain tends to be larger in elements with more dissimilar neighborhoods (higher $N^{\text {dissimilar}) . ~}$ This is true for the accumulated plastic strain at $166 \mathrm{~K}$ of the first cycle (point A**, Figure 3(b)) as well as for that at the start of the second cycle. This supports the hypothesis that the stress field due to incompatibilities at interfaces is responsible for austenite slip activity. These incompatibilities could arise at various types of interfaces - isolated A-M interfaces, impinging A-M interfaces or twinned M-M interfaces in contact with austenite. Figure 7 suggests that the latter two are the probable causes of slip. The standard deviation in the plastic strain (red curves) in Figure 7 is large. This suggests that a higher density of interfaces does not guarantee larger plastic strain generation. This can be rationalized on the basis of the rate-dependent plasticity. The rate-dependence implies that a larger plastic strain is generated in regions where the resolved shear stress on slip systems is large and extends over a longer time, compared to regions that also have a large stress but for a shorter period of time. A consequence is that larger plastic strain is expected in regions through which incompatible interfaces move slowly or arrest. In principle, the nature of the interface (e.g., ACV1 vs. A-CV2 vs. CV1-CV2) is expected to affect the distribution of resolved stress and thus the plastic flow on candidate slip systems. It should be noted that the rate dependence of slip is potentially exaggerated because of the relatively large rate exponent $(m)$ in the crystal plasticity law (Table 3). 

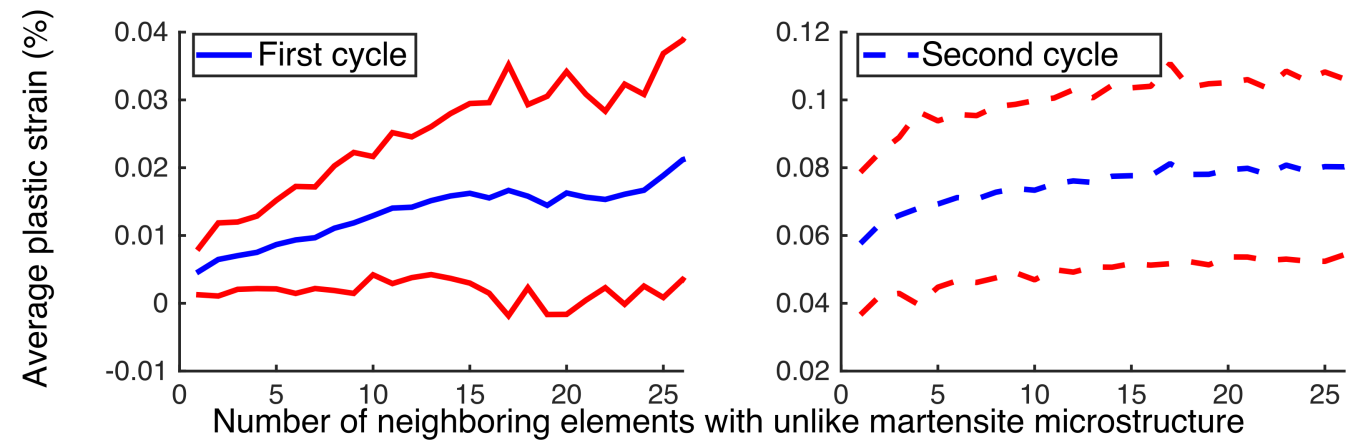

FIGURE 7 Plot of the average plastic strain vs. the number of neighbors of an element with dissimilar martensite microstructure as the parent. To calculate the quantity on the $\mathrm{X}$ axis, each element in the model is assigned a microstructure type, based on the dominant martensite CV formed during the first cycle and then, for a 26 element neighborhood of each element, the number of neighbors that did not form the same microstructure is counted. To calculate the quantity on the Y axis, the plastic strain is averaged over all elements that have the same number of dissimilar neighbors. The blue curve shows the mean and the red curves show the standard deviation. (a) Averaged plastic strain at $166 \mathrm{~K}$ of the $1^{\text {st }}$ cycle. (b) Plastic strain at the start of the $2^{\text {nd }}$ cycle. 
It is hypothesized that the different martensite microstructures in the load-biased vs. load-free cases will bias the activity on slip systems. Figure 8(a) shows the load-free sample, just after completion of the A-M transformation during the first cycle (point $\mathrm{A}^{* *}$, Figure 3(b)). All three CVs are present in the microstructure. Figure 8(b) shows the corresponding state in the load-biased case (point E', Figure 5(b)). Only CV1 and CV3 are present. This implies that the load-biased case has a reduced set of all possible M-M and potentially A-M interfaces. Table 4 partitions the top 1000 plastically deformed elements in the models according to the dominant slip system. The slip system with the largest equivalent plastic strain at the end of the first cycle is considered dominant. For the load-free case, the dominance is spread evenly among the six candidate austenite slip systems. For the load-biased case, the $\left(\begin{array}{lll}1 & 0 & 1\end{array}\right)\left[\begin{array}{lll}0 & 1 & 0\end{array}\right]$ and $\left(\begin{array}{lll}1 & 0 & \overline{1}\end{array}\right)\left[\begin{array}{lll}0 & 1 & 0\end{array}\right]$ systems are dominant in $\sim 88 \%$ of the elements. The bias stress produces zero resolved shear stress on all six slip systems. Thus, any differences in slip activity arise from the influence of martensite microstructure.

TABLE 4 Number of elements with a particular slip system being dominant at the end of first cycle. Top 1000 elements on the basis of plastic strain are considered.

\begin{tabular}{ccc}
\hline & $\begin{array}{c}\text { Elements with the corresponding slip system } \\
\text { being the dominant }\end{array}$ \\
\hline Slip system & Load-free & Load-biased \\
\hline 1 & 158 & 21 \\
2 & 204 & 19 \\
3 & 166 & 412 \\
4 & 150 & 466 \\
5 & 148 & 46 \\
6 & 175 & 37 \\
\hline
\end{tabular}

A small amount of martensite is present at the end of the heating cycle in all four simulations, irrespective of the differences in slip activity during the first cycle. This can be rationalized based on the form of the Landau polynomial-based energy. When the material point is near-austenitic $\left(v_{t} \rightarrow\right.$ 0 ), the slope of the Landau polynomial approaches zero (Figure 1). Thus, the driving force to complete the transformation is relatively small. Therefore, some residual martensite is expected to exist in simulations that span a finite amount of time. For the load-biased case, the biasing stress generates an additional driving force to form martensite. Irrespective of the temperature, the biasing stress is expected to stabilize a small fraction of martensite. This is present at the start and the end of the load-biased thermal cycles.

\subsection{Temporal Features of Martensite and Slip Evolution}

The martensite microstructure formed in the second cycle of the load-free case is more fragmented compared to the first cycle. Figures $8(\mathrm{a}, \mathrm{c})$ show the microstructure just after completion of the A-M transformation in the first vs. second cycles (points A** vs C', Figure $3(\mathrm{~b})$ ). The width of martensite bands is narrower in the second cycle. Consequently, there are more interfaces present at this stage. This has a consequence in terms of plasticity generation in the second cycle vs. first cycle. 


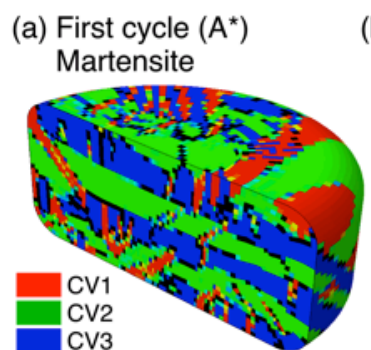
(b) First cycle $\left(A^{*}\right)$ Slip

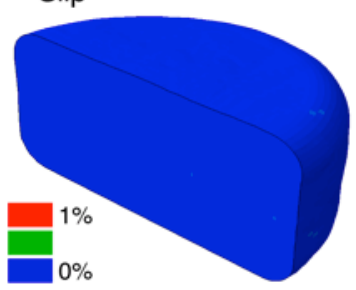
Martensite

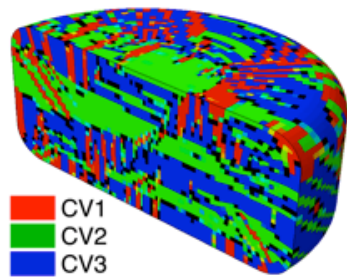

Bottom row - Load-biased thermal cycling (f) First cycle, cooling (E) Slip

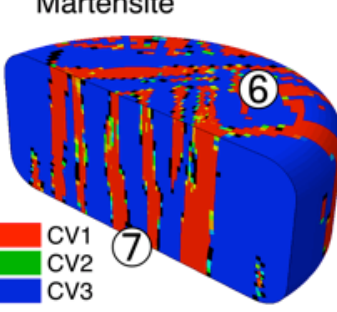

(g) First cycle, heating Martensite

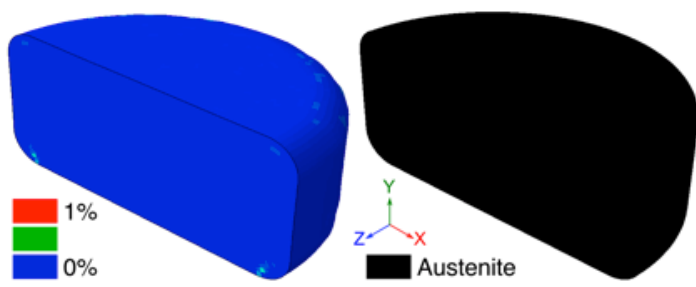

(d) Second cycle (C) Slip

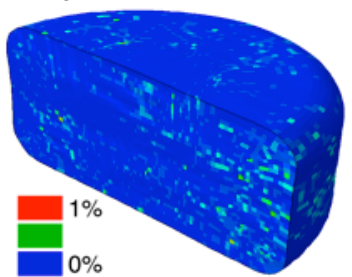

(S) (h) First cycle, heating (S) Slip

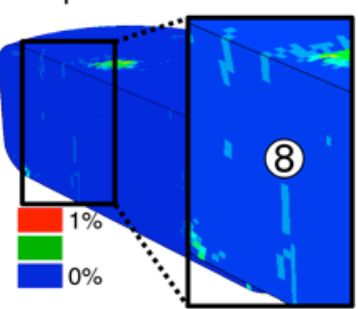

FIGURE 8 (a, c, e, g) Martensite and (b, d, f, h) plasticity at key points on the thermal cycling curve. (a, b) Martensite microstructure and plastic strain respectively at $166 \mathrm{~K}$ of the first cycle (point $\mathrm{A}^{* *}$ ) for the load-free simulation. (b, d) At $150 \mathrm{~K}$ of the second cycle (point $\mathrm{C}^{\prime}$ ) for the load-free simulation. (e, f) At $150 \mathrm{~K}$ (point E) for load-biased thermal cycling (g, h) At the end of first cycle (point S). 
It is hypothesized that the larger plastic strain in the second (vs. first) load-free cycle arises from the finer martensite and greater density of incompatible interfaces in the second cycle. Figures 8(a, b) show the martensite microstructure and slip distribution, respectively, just after the single crystal has fully transformed to martensite during the first cycle (point $\mathrm{A}^{* *}$, Figure $3 \mathrm{~b}$ ). The red, green and blue bands in Figure 8(a) depict regions dominated by CV1, CV2 and CV3, respectively. Figure 8(b) shows contours of equivalent plastic strain, with blue $=0 \%$ plastic strain and red $=1 \%$ plastic strain. The plastic deformation is small and the predicted plastic strain distribution is blue. For comparison, Figures $8(\mathrm{c}, \mathrm{d})$ show the corresponding plots just after the single crystal has fully transformed to martensite during the second cycle (point C', Figure $3 \mathrm{~b}$ ). The martensite CV band thickness is smaller and the slip activity is distributed over a larger volume compared to the first cycle. Also, the average plastic strain generation is greater in the second (0.1\%) vs. first $(0.02 \%)$ cycle (Figure $4(a)$ ). This supports the hypothesis that the stress field of martensite CV bands drives slip activity in austenite. The smaller CV band thickness in the second cycle produces a greater density of moving martensite interfaces and greater slip activity.

For the load-biased case, more plastic strain accumulates during the heating portion relative to the cooling portion. Figures $8(\mathrm{e}, \mathrm{f})$ show the martensite microstructure and equivalent plastic strain just after the first cooling portion (pt. E', Figure 5(b)). The distribution of slip activity does not show any specific pattern. Figure $8(\mathrm{~g}, \mathrm{~h})$ show the same quantities at the end of the first heating portion. At this point, all martensite has transformed back to austenite (black regions). The slip distribution here reflects a vertical pattern. The inset in Figure 8(h) shows the details of the slip pattern. These vertical bands of slip correspond spatially to the M-M interfaces shown in Figure 8(e). The absence of substantial slip after the first cooling portion (Figure 8(f)) and the presence of bands of plasticity after the first heating portion (Figure $8(\mathrm{~h})$ ) suggest that for the load-bias case, plastic deformation is caused by the motion of incompatible interfaces during heating, when martensite transforms to austenite.

The simulations predict that the evolution of slip varies substantially between material points. This is expected from the varied nature and speed of the martensite fronts as they pass through a material point. Figure 9 shows the local equivalent plastic strain as a function of local martensite volume fraction for nine representative elements in the load-free simulations. These elements are randomly selected from the top 1000 plastically-deformed elements. Two features stand out. First, seven of the nine points show that the plastic strain that accumulates during one portion (e.g., cooling) can be partially reversed during another portion (e.g., heating). This suggests that these material points experience a large enough reverse stress to change the direction of slip activity. This might arise from a change in stress field generated by an evolution in the local martensite microstructure. Second, three of the nine elements $(a, d, h)$ achieve the largest increments in plastic strain during cooling in the second cycle rather than heating (e.g., (e)). 
(a)
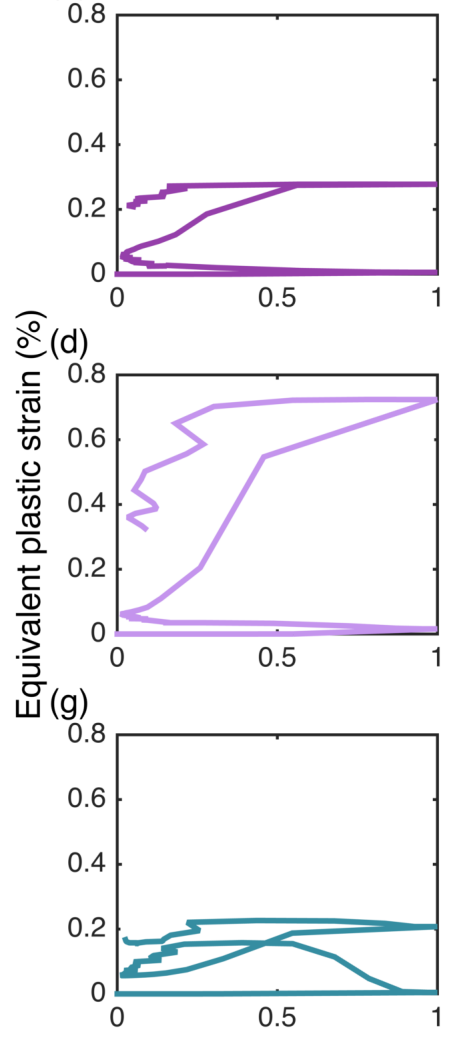

(b)

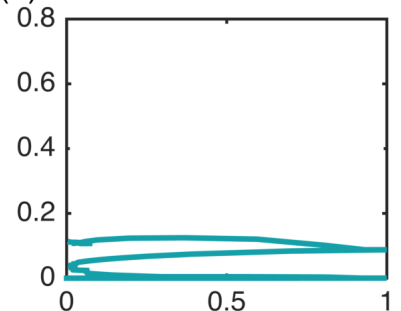

(e)

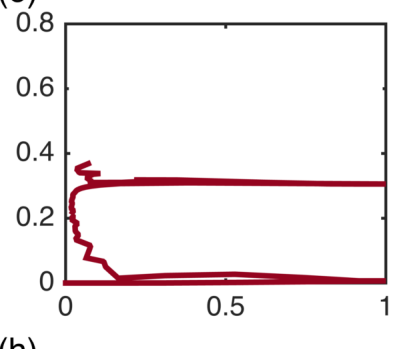

(h)

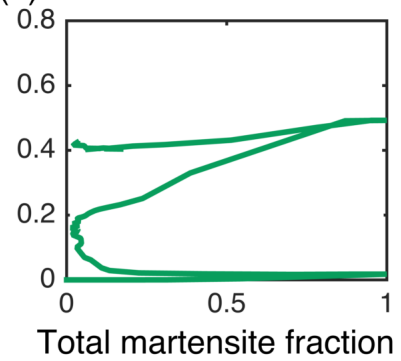

(c)

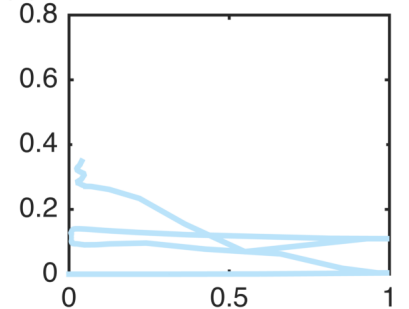

(f)

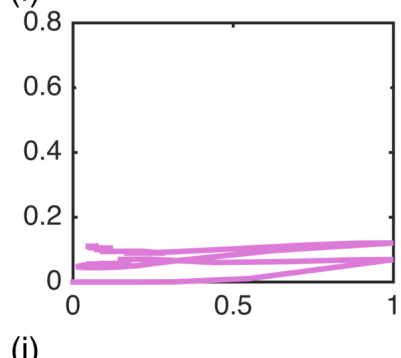

(i)

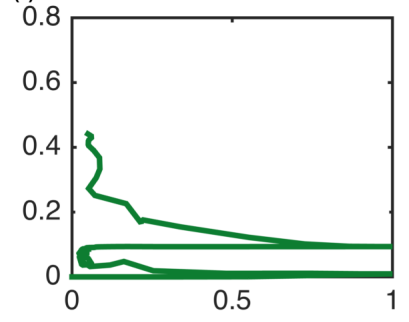

FIGURE 9 Plot of local equivalent plastic strain vs. local martensite volume fraction for nine representative elements in the load-free thermal cycling with-plasticity simulation. The elements were randomly chosen from top 1000 plastically deformed elements at the end of the second cycle. Three of the nine elements show significant plastic deformation during cooling of the second cycle. Seven of the elements show reverse slip. 
Plastic strain accumulation during the first cycle is hypothesized to aid the A-M transformation in the second cycle in two ways. First, plasticity generates internal stress in austenite that can increase the driving force for the start of the A-M transformation ( $f_{\mathrm{t}}$ in Eq. 6). Second, plasticity can relieve incompatibilities between interfaces that impinge near the end of the A-M transformation. Figures $10(\mathbf{a}, \mathbf{b})$ show the respective histograms for the martensite-start $\left(\theta_{\mathrm{MS}}\right)$ and martensite-finish $\left(\theta_{\mathrm{MF}}\right)$ temperatures, as measured at material points in load-free cases. $\theta_{\mathrm{MS}}$ and $\theta_{\mathrm{MF}}$ are defined as the temperatures at which the total martensite fraction at a material point reaches $20 \%$ and $80 \%$, respectively. During the first cycle, $\theta_{\mathrm{MS}} \sim 166 \mathrm{~K}$ for all material points in both the without and withplasticity cases, and $\theta_{\mathrm{MF}}$ ranges between 150 to $158 \mathrm{~K}$. However, the distribution in Figure 10(b) does not include all elements since some did not transform at the end of the first cooling cycle $(\theta=$ $150 \mathrm{~K}$ ). Figures (c, d) show the corresponding results for the second cycle. Both $\theta_{\mathrm{MS}}$ and $\theta_{\mathrm{MF}}$ are broader and shifted upward. The upward shift in $\theta_{\mathrm{MF}}$ is greater for the with-vs. without-plasticity cases. This supports the hypothesis that plastic deformation can assist the completion of the A-M transformation by accommodating incompatibilities between impinging martensite bands. The small difference in $\theta_{\mathrm{MS}}$ for the with- and without-plasticity simulations suggests that the small amount of plastic strain in the first cycle has a negligible effect on the start of transformation. The upward shift in $\theta_{\mathrm{MS}}$ and $\theta_{\mathrm{MF}}$ for both cases suggests that the small fraction of untransformed martensite at the end of the first cycle potentially aids the nucleation and growth of new martensite. These results include all $\left(1.14 \times 10^{5}\right)$ elements in the model. Most have very small $(0.08 \%)$ plastic strain at the end of the first cycle. A different trend in transformation temperature is revealed when only the elements with the largest plastic deformation are considered.

Large plastic deformation is hypothesized to potentially hinder transformation. Figures 10 (e-h) show $\theta_{\mathrm{MS}}$ and $\theta_{\mathrm{MF}}$ for the 1000 most plastically deformed elements in the model. Although plasticity does not alter the first-cycle values, plasticity does decrease the second-cycle values. This can be rationalized in terms of the stress relaxation caused by plastic deformation. Since plasticity is a stress relaxation mechanism, it can potentially reduce the available driving force for transformation. In principle, this can hinder the onset and completion of the A-M transformation. This effect is emphasized because only those elements with the largest plastic deformation were considered. Also, the reduced driving force for transformation caused by slip-enabled relaxation is expected to broaden the temperature range for $\theta_{\mathrm{MF}}$. This is observed qualitatively in Figure $10(\mathrm{~h})$.

Regions with larger plastic strain collectively have a wider transformation temperature range. Figure 6(c) shows the contour plots of the difference, $\Delta \theta=\theta_{\mathrm{MS}}-\theta_{\mathrm{MF}}$, for the second cycle of the load-free plasticity case. On average, $\Delta \theta=15 \mathrm{~K}$. However, $\Delta \theta$ is larger for elements located where M-M interfaces were present after completion of the A-M transformation in the first cycle (point $\mathrm{A}^{* *}$, Figure 3(b)). These elements also have larger plastic strains as shown in Figure 6(b) and discussed in Section 5.1. This supports the hypothesis that plastic strain is a competitive inelastic mechanism and it can reduce the local driving force for transformation. 
(c)

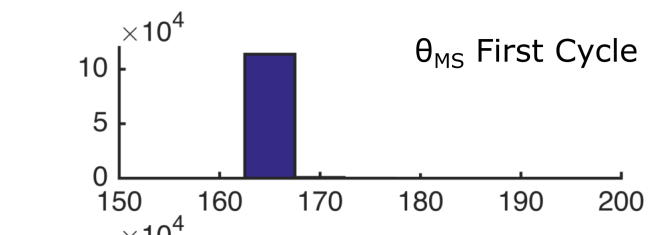

c)

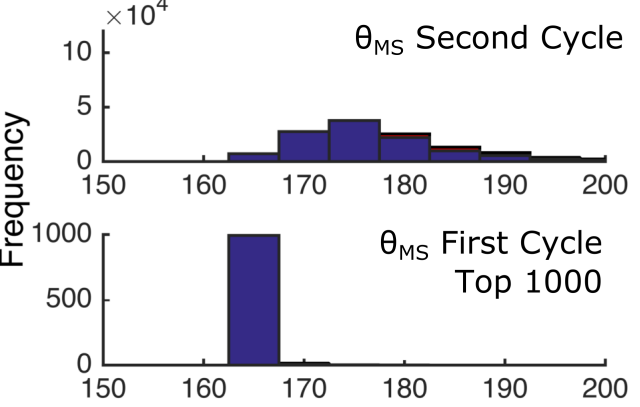

(g)

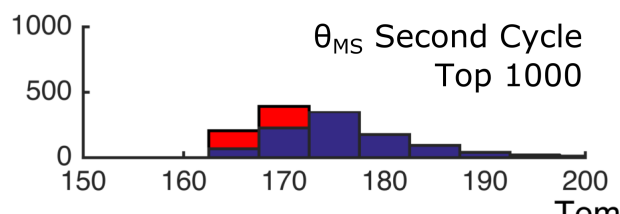

Temperature

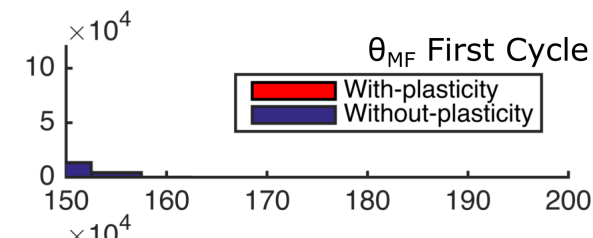

(b)

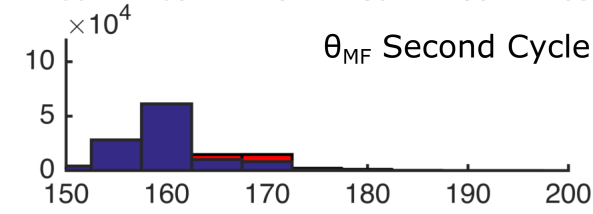

(d)
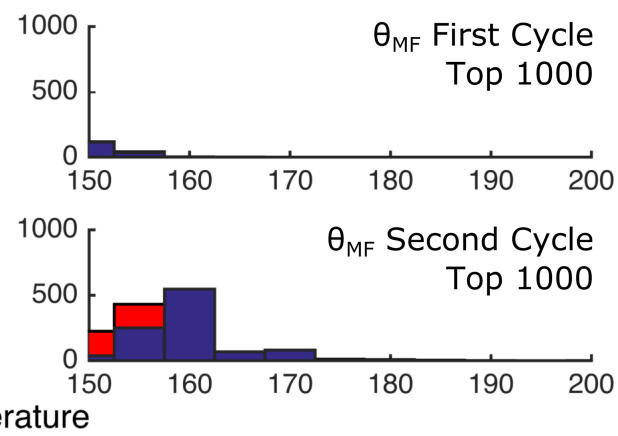

(h)

FIGURE 10 Histograms showing the transformation temperatures for the material points in the loadfree thermal cycling specimen. Plots in (a,b, e, f) are for all material points in the model. Plots (c, d) and $(\mathrm{g}, \mathrm{h})$ are similar to the plots $(\mathrm{a}, \mathrm{b}, \mathrm{e}, \mathrm{f})$ except, only top 1000 highly plastically deformed points are selected based on the plastic strains at the end of the second cycle. 


\section{Conclusions}

This work introduces a large-deformation finite element formalism to study the coupling of phase transformation and plasticity in shape memory alloys. Similar to many existing spectral approaches, the phase field method is used to model phase transformation at the martensite CV scale. This driving force includes contributions from the local stress state, chemical free energy changes between the austenite and martensite phases, and an interfacial energy. Similar to many existing finite element approaches, crystal slip in the austenite phase is modeled using a rate-dependent crystal plasticity law. The combination of phase transformation and crystal plasticity in a finite element solver provides a robust framework to examine complex geometries, e.g., polycrystalline constraints, crack tips, indentation geometries. It also captures large deformation features that accompany transformation and plasticity, including large rotation of material elements, orientations of A-M interfaces, and roughening of surfaces caused by these inelastic mechanisms.

A series of single crystal, load-free and load-biased thermal cycling simulations with cubic $\leftrightarrow$ tetragonal transformation systems and six $\left\{\begin{array}{lll}1 & 1 & 0\end{array}\right\}$ type austenite slip systems are carried out. The results are used to study key questions concerning the coupling between the transformation and plasticity in austenite.

1. Where does plasticity occur?

The local incompatibility at A-M interfaces is observed to drive plasticity in austenite. On average, the equivalent plastic strain tends to be larger at material points near $\mathrm{M}-\mathrm{M}$ interfaces. Among such points, rate-dependent plasticity is expected to increase where A-M interfaces arrest or propagate more slowly. Also, different A-M interfaces are observed to drive different austenite slip systems. Thus, textured martensite that is formed during cooling under load is observed to generate different slip system activity than untextured martensite formed during cooling without load.

2. When does plasticity occur?

The largest rate of change of average equivalent plastic strain with austenite volume fraction occurs when the austenite volume fraction $>80 \%$. Among the imposed heating and cooling portions, the plastic strain increment is relatively small during the first cooling portion. The increase in subsequent portions is attributed to an observed refinement in the martensite microstructure with cycling. At the local scale, the equivalent plastic strain at material points is observed to vary substantially and in some cases, it increases during cooling and decreases during heating, due to the complex stress history as A-M interfaces sweep forward and backward during a full thermal cycle. The resolved shear stress on a slip system can reverse sign with position along the interface (Bowers et al., 2014; Norfleet et al., 2009).

3. What is the effect of plasticity on the transformation in the subsequent cycles? Plasticity is observed to have different effects on transformation temperatures, depending on the relative magnitude of plastic strain. On the macroscopic level, $\theta_{\mathrm{MS}}$ changes negligibly during stress-free thermal cycling but $\theta_{\mathrm{MF}}$ shifts to higher values. This suggests that plastic strain aids the completion of the A-M transformation - by accommodating incompatibilities in the last regions to transform. On the local level, plasticity decreases both $\theta_{\mathrm{MS}}$ and $\theta_{\mathrm{MF}}$ at material points where the plastic strain is relatively large (the top 1000 out of $1.14 \times 10^{5}$ elements). Thus, the A-M transformation is hindered in regions with relatively large plasticity and aided in regions with relatively little plasticity.

The finite deformation phase field/crystal plasticity simulations couple with novel experimental techniques, including recent TEM observations showing that specific A-M interfaces active select 
austenite slip systems (Bowers et al., 2014). The simulations can be used to interpret novel high energy x-ray diffraction-based techniques that measure deformation at the grain scale (Bernier et al., 2011; Suter et al., 2006). In principle, the formalism presented here can be applied to a variety of systems that exhibit phase transformations, plasticity, and even twinning.

Some qualifications and attributes to the model and numerical approach include the nature of the plasticity and the explicit finite element formulation. The present continuum plasticity formulation, although efficient, assigns a rate-dependent flow strength that is independent of material scale. At the nm-scale of martensite structures, the ability to plastically deform may be controlled by the proximity of dislocation sources either within A-M interfaces or in nearby highly stressed austenite regions. Remarkably, experiments in NiTi shape memory alloys show numerous dislocation loops $\sim 10 \mathrm{~s}$ of $\mathrm{nm}$ in dimension, suggesting that dislocation sources exist at the finest nm scales (Norfleet et al., 2009). The explicit finite element approach is appealing in that analytic versions of Jacobians are not required compared to implicit formulations. However, computational times reach $\sim 12 \mathrm{hrs}$ with a parallelized code running on several processors- considerably less efficient than spectral methods (Jin et al., 2001; Wang and Khachaturyan, 1997). These concerns are offset by the ability to capture the kinematics of large deformation and a variety of model geometries.

\section{ACKNOWLEDGEMENTS}

HP acknowledges the support of the National Science Foundation (Award DMR 0907561) and PA acknowledges the support of the DOE Office of Basic Energy Sciences (Grant SC-0001258). The support of the Ohio Supercomputer Center (Grant PAS0676) is greatly appreciated for execution of the simulations.

\section{REFERENCES}

ABAQUS, 2008. . Abaqus Ref. Man.

Ammar, K., Appolaire, B., Cailletaud, G., Forest, S., 2009. Combining phase field approach and homogenization methods for modelling phase transformation in elastoplastic media. Eur. J. Comput. Mech. 18, 485-523. doi:10.3166/ejcm.18.485-523

Anand, L., 2012. A Cahn-Hilliard-type theory for species diffusion coupled with large elastic-plastic deformations. J. Mech. Phys. Solids 60, 1983-2002. doi:10.1016/j.jmps.2012.08.001

Artemev, A., Jin, Y., Khachaturyan, A.G., 2001. Three-dimensional phase field model of proper martensitic transformation. Acta Mater. 49, 1165-1177. doi:10.1016/s1359-6454(01)00021-0

Artemev, A., Wang, Y., Khachaturyan, A.G., 2000. Three-dimensional phase field model and simulation of martensitic transformation in multilayer systems under applied stresses. Acta Mater. 48, 2503-2518.

Auricchio, F., Fugazza, D., DesRoches, R., 2008. Rate-dependent thermo-mechanical modelling of superelastic shape-memory alloys for seismic applications. J. Intell. Mater. Syst. Struct. 19, 47-61. doi:10.1177/1045389X06073426

Auricchio, F., Petrini, L., 2004. A three-dimensional model describing stresstemperature induced solid phase transformations: solution algorithm and 
boundary value problems. Int. J. Numer. Methods Eng. 61, 807-836. doi:10.1002/nme.1086

Ball, J.M., James, R.D., 1987. FINE PHASE MIXTURES AS MINIMIZERS OF ENERGY. Arch. Ration. Mech. Anal. 100, 13-52. doi:10.1007/bf00281246

Benafan, O., Noebe, R.D., Padula, S.A., Brown, D.W., Vogel, S., Vaidyanathan, R., 2014. Thermomechanical cycling of a NiTi shape memory alloy-macroscopic response and microstructural evolution. Int. J. Plast. 56, 99-118. doi:10.1016/j.ijplas.2014.01.006

Bernier, J.V., Barton, N.R., Lienert, U., Miller, M.P., 2011. Far-field high-energy diffraction microscopy: a tool for intergranular orientation and strain analysis. J. Strain Anal. Eng. Des. 0309324711405761. doi:10.1177/0309324711405761

Bhattacharya, K., 2003. Microstructure of Martensite: Why it forms and how it gives rise to the shape-memory effect. Oxford University Press.

Bowers, M.L., Chen, X., De Graef, M., Anderson, P.M., Mills, M.J., 2014. Characterization and modeling of defects generated in pseudoelastically deformed NiTi microcrystals. Scr. Mater. 78-79, 69-72. doi:10.1016/j.scriptamat.2014.02.001

Chen, Y., Schuh, C.A., 2015. A coupled kinetic Monte Carlo-finite element mesoscale model for thermoelastic martensitic phase transformations in shape memory alloys. Acta Mater. 83, 431-447. doi:10.1016/j.actamat.2014.10.011

Christ, D., Reese, S., 2008. Finite-element modelling of shape memory alloys-A comparison between small-strain and large-strain formulations. Mater. Sci. Eng. A, Proceedings of the 7th European Symposium on Martensitic Transformations, $\quad$ ESOMAT $2006 \quad 481-482, \quad 343-346$. doi:10.1016/j.msea.2006.11.174

Cisse, C., Zaki, W., Ben Zineb, T., 2016. A review of constitutive models and modeling techniques for shape memory alloys. Int. J. Plast. 76, 244-284. doi:10.1016/j.ijplas.2015.08.006

Cui, J., Shield, T.W., James, R.D., 2004. Phase transformation and magnetic anisotropy of an iron-palladium ferromagnetic shape-memory alloy. Acta Mater. 52, 3547. doi:10.1016/j.actamat.2003.08.024

Delville, R., Malard, B., Pilch, J., Sittner, P., Schryvers, D., 2011. Transmission electron microscopy investigation of dislocation slip during superelastic cycling of $\mathrm{Ni}$ Ti wires. Int. J. Plast. 27, 282-297. doi:10.1016/j.ijplas.2010.05.005

Eggeler, G., Hornbogen, E., Yawny, A., Heckmann, A., Wagner, M., 2004. Structural and functional fatigue of NiTi shape memory alloys. Mater. Sci. Eng. A 378, 24-33. doi:10.1016/j.msea.2003.10.327

Gao, Y., Zhou, N., Wang, D., Wang, Y., 2014. Pattern formation during cubic to orthorhombic martensitic transformations in shape memory alloys. Acta Mater. 68, 93-105. doi:10.1016/j.actamat.2014.01.012

Gurtin, M.E., 2000. On the plasticity of single crystals: free energy, microforces, plastic-strain gradients. J. Mech. Phys. Solids 48, 989-1036. doi:10.1016/S0022-5096(99)00059-9

Hamilton, R.F., Sehitoglu, H., Chumlyakov, Y., Maier, H.J., 2004. Stress dependence of the hysteresis in single crystal NiTi alloys. Acta Mater. 52, 3383-3402. 
Javanbakht, M., Levitas, V.I., 2015. Interaction between phase transformations and dislocations at the nanoscale. Part 2: Phase field simulation examples. J. Mech. Phys. Solids 82, 164-185. doi:10.1016/j.jmps.2015.05.006

Jin, Y.M., Artemev, A., Khachaturyan, A.G., 2001. Three-dimensional phase field model of low-symmetry martensitic transformation in polycrystal: simulation of $\zeta^{\prime} 2$ martensite in AuCd alloys. Acta Mater. 49, 2309-2320. doi:10.1016/S1359-6454(01)00108-2

Kalidindi, S.R., Bronkhorst, C.A., Anand, L., 1992. Crystallographic texture evolution in bulk deformation processing of fcc metals. J. Mech. Phys. Solids 40, 537569.

Kibey, S., Sehitoglu, H., Johnson, D.D., 2009. Energy landscape for martensitic phase transformation in shape memory NiTi. Acta Mater. 57, 1624-1629. doi:10.1016/j.actamat.2008.12.008

Kundin, J., Pogorelov, E., Emmerich, H., 2015. Numerical investigation of the interaction between the martensitic transformation front and the plastic strain in austenite. J. Mech. Phys. Solids 76, 65-83. doi:10.1016/j.jmps.2014.12.007

Kundin, J., Raabe, D., Emmerich, H., 2011. A phase-field model for incoherent martensitic transformations including plastic accommodation processes in the austenite. J. Mech. Phys. Solids 59, 2082-2102. doi:10.1016/j.jmps.2011.07.001

Lee, E.H., 1969. Elastic-plastic deformation at finite strains. J Appl Mech 36, 1-6.

Levitas, V.I., Javanbakht, M., 2015. Interaction between phase transformations and dislocations at the nanoscale. Part 1. General phase field approach. J. Mech. Phys. Solids 82, 287-319. doi:10.1016/j.jmps.2015.05.005

Levitas, V.I., Javanbakht, M., 2013. Phase field approach to interaction of phase transformation and dislocation evolution. Appl. Phys. Lett. 102, 251904. doi:10.1063/1.4812488

Levitas, V.I., Preston, D.L., 2002. Three-dimensional Landau theory for multivariant stress-induced martensitic phase transformations. I. Austenite <-> martensite. Phys. Rev. B 66, 9. doi:10.1103/PhysRevB.66.134206

Liu, L., Ding, X., Li, J., Lookman, T., Sun, J., 2014. Direct observation of hierarchical nucleation of martensite and size-dependent superelasticity in shape memory alloys. Nanoscale 6, 2067-2072.

Malik, A., Amberg, G., Borgenstam, A., Ågren, J., 2013. Phase-field modelling of martensitic transformation: the effects of grain and twin boundaries. Model. Simul. Mater. Sci. Eng. 21, 085003. doi:10.1088/0965-0393/21/8/085003

Manchiraju, S., Anderson, P.M., 2010. Coupling between martensitic phase transformations and plasticity: A microstructure-based finite element model. Int. J. Plast. 26, 1508-1526. doi:10.1016/j.ijplas.2010.01.009

Manchiraju, S., Gaydosh, D., Benafan, O., Noebe, R., Vaidyanathan, R., Anderson, P.M., 2011. Thermal cycling and isothermal deformation response of polycrystalline NiTi: Simulations vs. experiment. Acta Mater. 59, 5238-5249. doi:10.1016/j.actamat.2011.04.063 

Scr. Metall. 17, 1167-1171. doi:10.1016/0036-9748(83)90277-6

Mohd Jani, J., Leary, M., Subic, A., Gibson, M.A., 2014. A review of shape memory alloy research, applications and opportunities. Mater. Des. 56, 1078-1113. doi:10.1016/j.matdes.2013.11.084

Morrison, K.R., Cherukara, M.J., Kim, H., Strachan, A., 2015. Role of grain size on the martensitic transformation and ultra-fast superelasticity in shape memory alloys. Acta Mater. 95, 37-43. doi:10.1016/j.actamat.2015.05.015

Norfleet, D.M., Sarosi, P.M., Manchiraju, S., Wagner, M.F.X., Uchic, M.D., Anderson, P.M., Mills, M.J., 2009. Transformation-induced plasticity during pseudoelastic deformation in Ni-Ti microcrystals. Acta Mater. 57, 35493561. doi:10.1016/j.actamat.2009.04.009

Pan, H., Thamburaja, P., Chau, F.S., 2007. Multi-axial behavior of shape-memory alloys undergoing martensitic reorientation and detwinning. Int. J. Plast. 23, 711-732.

Peirce, D., Asaro, R.J., Needleman, A., 1982. An analysis of nonuniform and localized deformation in ductile single crystals. Acta Metall. 30, 1087-1119.

Pelton, A.R., Huang, G.H., Moine, P., Sinclair, R., 2012. Effects of thermal cycling on microstructure and properties in Nitinol. Mater. Sci. Eng. A 532, 130-138.

Polatidis, E., Zotov, N., Bischoff, E., Mittemeijer, E.J., 2015. The effect of cyclic tensile loading on the stress-induced transformation mechanism in superelastic NiTi alloys: an in-situ X-ray diffraction study. Scr. Mater. 100, 59-62. doi:10.1016/j.scriptamat.2014.12.013

Reese, S., Christ, D., 2008. Finite deformation pseudo-elasticity of shape memory alloys - Constitutive modelling and finite element implementation. Int. J. Plast. 24, 455-482. doi:10.1016/j.ijplas.2007.05.005

Richards, A.W., Lebensohn, R.A., Bhattacharya, K., 2013. Interplay of martensitic phase transformation and plastic slip in polycrystals. Acta Mater. 61, 43844397. doi:10.1016/j.actamat.2013.03.053

Stebner, A.P., Brinson, L.C., 2013. Explicit finite element implementation of an improved three dimensional constitutive model for shape memory alloys. Comput. Methods Appl. Mech. Eng. 257, 17-35. doi:10.1016/j.cma.2012.12.021

Suter, R.M., Hennessy, D., Xiao, C., Lienert, U., 2006. Forward modeling method for microstructure reconstruction using x-ray diffraction microscopy: Singlecrystal verification. Rev. Sci. Instrum. 77, 123905. doi:10.1063/1.2400017

Tadaki, T., Shimizu, K., 1975. High tetragonality of the thermoelastic Fe3Pt martensite and small volume change during the transformation. Scr. Metall. 9, 771-776. doi:10.1016/0036-9748(75)90238-0

Thamburaja, P., 2010. A finite-deformation-based phenomenological theory for shape-memory alloys. Int. J. Plast. 26, 1195-1219. doi:10.1016/j.ijplas.2009.12.004

Thamburaja, P., 2005. Constitutive equations for martensitic reorientation and detwinning in shape-memory alloys. J. Mech. Phys. Solids 53, 825-856. 
Thamburaja, P., Anand, L., 2001. Polycrystalline shape-memory materials: Effect of crystallographic texture. J. Mech. Phys. Solids 49, 709-737.

Tseng, L.W., Ma, J., Wang, S.J., Karaman, I., Kaya, M., Luo, Z.P., Chumlyakov, Y.I., 2015. Superelastic response of a single crystalline FeMnAlNi shape memory alloy under tension and compression. Acta Mater. 89, 374-383. doi:10.1016/j.actamat.2015.01.009

Wang, Y., Khachaturyan, A.G., 1997. Three-dimensional field model and computer modeling of martensitic transformations. Acta Mater. 45, 759-773.

Yamanaka, A., Takaki, T., Tomita, Y., 2010. Elastoplastic phase-field simulation of martensitic transformation with plastic deformation in polycrystal. Int. J. Mech. Sci. 52, 245-250. doi:10.1016/j.ijmecsci.2009.09.020

Yamanaka, A., Takaki, T., Tomita, Y., 2008. Elastoplastic phase-field simulation of self- and plastic accommodations in martensitic transformation. Mater. Sci. Eng. A 491, 378-384. doi:10.1016/j.msea.2008.02.035

Yu, C., Kang, G., Kan, Q., 2015a. A micromechanical constitutive model for anisotropic cyclic deformation of super-elastic NiTi shape memory alloy single crystals. J. Mech. Phys. Solids 82, 97-136. doi:10.1016/j.jmps.2015.05.012

Yu, C., Kang, G., Kan, Q., Song, D., 2013. A micromechanical constitutive model based on crystal plasticity for thermo-mechanical cyclic deformation of NiTi shape memory alloys. Int. J. Plast. 44, 161-191. doi:10.1016/j.ijplas.2013.01.001

Yu, C., Kang, G., Song, D., Kan, Q., 2015b. Effect of martensite reorientation and reorientation-induced plasticity on multiaxial transformation ratchetting of super-elastic NiTi shape memory alloy: New consideration in constitutive model. Int. J. Plast. 67, 69-101. doi:10.1016/j.ijplas.2014.10.001

Zaki, W., Moumni, Z., 2007. A 3D model of the cyclic thermomechanical behavior of shape memory alloys. J. Mech. Phys. Solids 55, 2427-2454.

Zambaldi, C., Yang, Y., Bieler, T.R., Raabe, D., 2012. Orientation informed nanoindentation of $\alpha$-titanium: Indentation pileup in hexagonal metals deforming by prismatic slip. J. Mater. Res. 27, 356-367. doi:10.1557/jmr.2011.334 
TABLE 1 Cubic to tetragonal transformation strain matrices for the three CVs. Below each matrix, a graphical representation of the deformation induced is shown.

$E_{1}=\left(\begin{array}{ccc}-0.03 & 0 & 0 \\ 0 & -0.03 & 0 \\ 0 & 0 & 0.06\end{array}\right) \quad E_{2}=\left(\begin{array}{ccc}-0.03 & 0 & 0 \\ 0 & 0.06 & 0 \\ 0 & 0 & -0.03\end{array}\right) \quad E_{3}=\left(\begin{array}{ccc}0.06 & 0 & 0 \\ 0 & -0.03 & 0 \\ 0 & 0 & -0.03\end{array}\right)$


TABLE 2 Cubic slip systems modeled in the austenite phase.

\begin{tabular}{ccc}
\hline Slip system & Slip plane & Burgers vectors \\
\hline 1 & $\left(\begin{array}{lll}0 & 1 & 1\end{array}\right)$ & {$\left[\begin{array}{lll}1 & 0 & 0\end{array}\right]$} \\
2 & $\left(\begin{array}{lll}0 & 1 & \overline{1}\end{array}\right)$ & {$\left[\begin{array}{lll}1 & 0 & 0\end{array}\right]$} \\
3 & $\left(\begin{array}{lll}1 & 0 & 1\end{array}\right)$ & {$\left[\begin{array}{lll}0 & 1 & 0\end{array}\right]$} \\
4 & $\left(\begin{array}{llll}1 & 0 & \overline{1}\end{array}\right)$ & {$\left[\begin{array}{lll}0 & 1 & 0\end{array}\right]$} \\
5 & $\left(\begin{array}{lll}1 & 1 & 0\end{array}\right)$ & {$\left[\begin{array}{lll}0 & 0 & 1\end{array}\right]$} \\
6 & $\left(\begin{array}{llll}1 & 1 & 0\end{array}\right)$ & {$\left[\begin{array}{lll}0 & 0 & 1\end{array}\right]$}
\end{tabular}


TABLE 3 Material properties used in the thermal cycling simulations.

\begin{tabular}{|c|c|}
\hline Elastic and thermal & $\begin{array}{l}\text { Elastic constants }(\mathrm{GPa}): C_{11(\mathrm{~A})}=130 ; C_{22(\mathrm{~A})}=98 ; C_{44(\mathrm{~A})}=21 ; C_{\mathrm{M}}=C_{\mathrm{A}} / 2 \\
\text { Thermal expansion coefficients }\left(10^{-6} / \mathrm{K}\right): \alpha_{\mathrm{A}}=11 ; \alpha_{\mathrm{M}}=6.6\end{array}$ \\
\hline Phase transformation & $\begin{array}{l}\text { Equilibrium transformation temperature }(\mathrm{K}): \theta_{\mathrm{T}}=250 \\
\text { Latent heat of transformation/unit volume }(\mathrm{MPa}): \lambda_{\mathrm{T}}=20 \\
\text { Landau polynomial coefficients: } p=1.0, q=-0.5, b=1.0, d=-2.0, \mathrm{f}=1.0 \\
\text { Mobility (/s): } M=3.5 \times 10^{-2} \\
\text { Number of martensite CVs: } N_{\mathrm{T}}=3 \\
\text { Interface energy term }(\mathrm{J} / \mathrm{m}), K_{t u}=0\end{array}$ \\
\hline Austenite plasticity & $\begin{array}{l}\text { Reference shear strain rate }(/ \mathrm{s}): \dot{\gamma}_{0}=0.02 \\
\text { Strain rate exponent: } \mathrm{m}=0.5 \\
\text { Initial slip system shear strength }(\mathrm{MPa}): g_{s}^{0}=160 \text { for the with-plasticity } \\
\text { case and } g_{s}^{0}=10^{6} \text { for the without-plasticity case. } \\
\text { Self-hardening coefficient (MPa): } h_{0}=1250 \\
\text { Ratio of self to latent hardening: } \mathrm{Q}=1.4 \\
\text { Hardening exponent: } \mathrm{a}=0.1 \\
\text { Saturation shear strength }(\mathrm{MPa}): g_{\mathrm{sat}}=1000 \\
\text { Number of slip systems: } N_{\mathrm{S}}=6\end{array}$ \\
\hline
\end{tabular}


TABLE 4 Number of elements with a particular slip system being dominant at the end of first cycle. Top 1000 elements on the basis of plastic strain are considered.

\begin{tabular}{ccc}
\hline & $\begin{array}{r}\text { Elements with the corresponding slip system } \\
\text { being the dominant }\end{array}$ \\
\hline Slip system & Load-free & Load-biased \\
\hline 1 & 158 & 19 \\
3 & 204 & 412 \\
4 & 166 & 466 \\
5 & 150 & 46 \\
6 & 148 & 37 \\
\hline
\end{tabular}

